\title{
Rasgos del liderazgo femenino en fundaciones del Valle del Cauca ${ }^{1}$
}

\author{
Beatriz Eugenia Agudelo-Orrego ${ }^{2}$ \\ Universidad del Valle \\ beatriz.agudelo@correounivalle.edu.co
}

\section{DOI: https://doi.org/10.21158/01208160.n91.2021.3025}

Fecha de recepción: 23 de julio de 2020

Fecha de aprobación: 21 de julio de 2021

Fecha de publicación: 01 de octubre de 2021

Cómo citar este artículo: Agudelo-Orrego, B. E. (2021). Rasgos del liderazgo femenino en fundaciones del Valle del Cauca. Revista Escuela de Administración de Negocios, (91), páginas. DOI: https://doi.org/10.21158/01208160.n91.2021.3025

\section{Resumen}

Esta investigación tuvo por objetivo analizar los rasgos del liderazgo en un grupo de mujeres directoras de 31 fundaciones en el Valle del Cauca, con el fin de establecer cuáles de estos predominan en las participantes y afectan, en mayor medida, la variación de la dimensión de la personalidad que los integra, para predecir su potencial de liderazgo. Se utilizó un enfoque cuantitativo haciendo uso de los procedimientos de la investigación descriptiva dirigidos a conocer cuáles de los rasgos poseen las directoras, y de la investigación correlacional con el propósito de identificar el impacto que estos tienen en la dimensión correspondiente. Para lograrlo, se aplicó una encuesta adaptada del inventario revisado de personalidad NEO PI-R compuesta por 25 descriptores, clasificados en cinco dimensiones: neurotismo, extroversión, apertura a nuevas experiencias, agradabilidad y escrupulosidad; cada una de estas abarcó cinco rasgos del liderazgo, que fueron analizados a partir de la escala diferencial semántica. El análisis de la información permitió establecer que un gran número de fundaciones participantes están ubicadas en Cali y se dedican a brindar atención integral a niños y jóvenes en situación de pobreza, violencia y abandono; la mayoría de las directoras tienen entre 41 y 60 años, no tienen un salario establecido y algunas son profesionales. Así mismo, se estableció que, en general, las directoras son especialmente extrovertidas, lo que repercute en su sociabilidad; agradables por la confianza que generan y tienen alta capacidad de logro, evidenciada en su deliberación, competencia y autodisciplina.

Palabras clave: liderazgo; liderazgo femenino; género y liderazgo; teoría de rasgos; rasgos de liderazgo; potencial de liderazgo; fundaciones sociales.

\footnotetext{
${ }^{1}$ Artículo derivado de los resultados preliminares de la tesis doctoral titulada «Liderazgo femenino en las fundaciones del Valle del Cauca», sustentada el 22 de marzo del 2018 para obtener el título de Doctor en Administración en la Universidad Autónoma de Querétaro en Querétaro, México.

2 Tecnóloga en Química - Universidad Tecnológica de Pereira. Administradora de Empresas - Universidad del Valle. Magíster en Administración de Empresas - Universidad del Valle. Especialista en Gerencia de Procesos de Calidad e Innovación - Universidad Ean. Doctora en Administración - Universidad Autónoma de Querétaro. ORCID: https://orcid.org/0000-0001-8579-5292
} 


\title{
Traits of Female Leadership in Foundations in Valle del Cauca
}

\begin{abstract}
This research aimed to analyze the leadership traits in a group of women directors of 31 foundations in Valle del Cauca in order to establish which of these traits predominate in the participants and affect, to a greater extent, the variation of the personality dimension that integrates them, in pursuance of predicting their leadership potential. The study uses a quantitative approach, applying descriptive research procedures to find out which of the traits the directors possess and correlational research to identify the impact these traits have on the corresponding dimension. To achieve this requires applying a survey adapted from the revised inventory of NEO PI-R personality, composed of 25 descriptors classified into five dimensions: neuroticism, extroversion, openness to new experiences, agreeableness, and conscientiousness. Each covered five leadership traits, which were analyzed based on the semantic differential scale. The analysis of the information made it possible to establish that a large number of the participating foundations are located in Cali and are dedicated to providing comprehensive care to children and young people in situations of poverty, violence, and abandonment. Most of the directors are between 41 and 60 years old, do not have a fixed salary, and some of them are professionals. Likewise, it was established that, in general, the directors are especially extroverted, which impacts their sociability; they are pleasant because of the trust they generate and have a high capacity for achievement, as evidenced in their deliberation, competence, and self-discipline.
\end{abstract}

Keywords: leadership; women's leadership; gender and leadership; trait theory; leadership traits; leadership potential; social foundations.

Traços da liderança feminina nas fundações do Vale do Cauca

\section{Resumo}

Esta pesquisa teve como objetivo analisar os traços de liderança em um grupo de mulheres que são diretoras em 31 fundações no Vale do Cauca, a fim de estabelecer quais desses traços predominam nas participantes e afetam, maiormente, a variação da dimensão da personalidade que os integra, para prever seu potencial de liderança. Utilizou-se uma abordagem quantitativa, recorrendo a procedimentos de pesquisa descritiva, com o objetivo de conhecer quais dos traços têm as diretoras, e de pesquisa correlacional, para identificar o impacto que eles têm na dimensão correspondente. Para tanto, aplicou-se um questionário adaptado do inventário revisado de personalidade NEO PI-R, composto por 25 descritores, classificados em cinco dimensões: neuroticismo, extroversão, abertura a novas experiências, afabilidade e escrupulosidade. Cada uma delas englobou cinco traços de liderança, que foram analisados por meio da escala de diferencial semântico. A análise da informação permitiu constatar que grande parte das fundações participantes estão localizadas em Cali e se dedicam a prestar atenção integral a crianças e jovens em situação de pobreza, violência e abandono. A maioria das diretoras tem entre 41 e 60 anos, não possui salário fixo e algumas são profissionais liberais. Da mesma forma, ficou estabelecido que, em geral, as diretoras são especialmente extrovertidas, o que tem repercussão em sua sociabilidade. São agradáveis pela confiança que geram e possuem alta capacidade de realização, evidenciada em sua deliberação, competência e autodisciplina.

Palavras-chave: liderança; liderança feminina; gênero e liderança; teoria de traços; traços de liderança; potencial de liderança; fundações sociais. 
Caractéristiques du leadership féminin dans les fondations du Valle del Cauca

\begin{abstract}
Résumé
L'objectif de cette étude consiste à analyser les traits du leadership féminin de 31 responsables de fondations de la région du Valle del Cauca pour établir les caractéristiques morales prédominantes des participantes ; caractéristiques affectant dans une plus large mesure des variations de dimension personnelle pour prédire le potentiel de leadership. Une approche quantitative a été mis en place avec des procédures descriptives visant à déceler les traits du leadership et des recherches corrélationnelles pour identifier l'impact sur les dimensions étudiées. Le groupe, qui se compose de 25 descripteurs, a été soumis à une étude de personnalité de type NEO PI-R classée en cinq dimensions : névrosisme, extraversion, ouverture à de nouvelles expériences, amabilité et conscience. Chaque descripteur comprend cinq traits du leadership, analysés à l'aide d' une échelle différentielle de sémantique. L'analyse des informations a permis d'établir qu'un grand nombre de fondations participantes se trouvent dans la ville de Cali et se consacrent à la prise en charge d'enfants et des jeunes en situation d'extrême pauvreté, de violence et d'abandon. La plupart des directrices de centre ont entre 41 et 60 ans, n'ont pas de salaire fixe et certaines ont une activité professionnelle distincte. L'étude révèle enfin que ces femmes sont généralement très extraverties, ce qui renforce leur sociabilité, compétentes, disciplinées, agréables et ont une grande capacité à tenir leurs objectifs.
\end{abstract}

Mots-clés: leadership; leadership féminin; genre et leadership; théorie des traits; traits de leadership; potentiel de leadership; fondements sociaux.

\title{
1. Introducción
}

Las organizaciones desempeñan un papel fundamental en la sociedad, dado que permiten dinamizar la economía a partir no solo de la producción de bienes y servicios, sino de la generación de empleo que redunda en el bienestar de la población. Así, surgen las organizaciones sin fines de lucro (OSFL), entre ellas las fundaciones, entidades que llevan a cabo acciones de beneficio propio o comunitario cuyo fin, en sí mismo, no contiene la obtención de ganancias meramente dinerarias, sino la resolución de necesidades del orden social y de otro tipo (Alcaldía de Bogotá, 2014).

El objeto social de estas organizaciones implica la interacción con diversos grupos, los llamados stakeholders, cuyas demandas deben ser satisfechas a partir de una efectiva gestión de los recursos escasos, es decir, del liderazgo de la gerencia. Este tema ha sido abordado desde diversas disciplinas para generar un sinnúmero de definiciones y teorías basadas en la capacidad del líder de interpretar el entorno y encauzar los esfuerzos de las personas a su cargo en la consecución de los objetivos de la organización. 
A fin de lograrlo, es importante reconocer que las organizaciones de hoy han cambiado, pues hay mayor presencia de mujeres, lo que se debe a muchas razones, entre las que se resalta la posibilidad que ahora tienen de acceder a la educación y su talento para ocupar cargos directivos (Cárdenas, 2002). Este talento se evidencia en el ejercicio de un liderazgo efectivo a partir de tres valores femeninos: ética de cuidado, comunicación informal interpersonal y dirección participativa (Vasada, 2012).

A partir de lo anterior, se observa que el liderazgo reúne una serie de atributos y habilidades, pero también estructuras cognitivas que permiten a la persona tener características de líder; de allí el interés por analizar dichas características.

En este sentido, este artículo presenta la caracterización de los rasgos del liderazgo de 31 mujeres que dirigen fundaciones localizadas en el Valle del Cauca, quienes se dedican a desarrollar proyectos de intervención dirigidos a atender a población en condición de vulnerabilidad.

Así, en primera instancia, se muestra una revisión de los referentes de esta investigación como lo son las teorías del liderazgo, el liderazgo en las OSFL y el género. En el apartado «metodología» se presenta la descripción de las fundaciones participantes, el detalle del instrumento utilizado, la forma de aplicación a sus directoras y la descripción de los análisis realizados. Luego se muestran los resultados obtenidos con referencia a los aspectos sociodemográficos de las fundaciones y sus directoras, correspondientes al análisis descriptivo que presenta los rasgos de liderazgo preponderantes en ellas y al análisis inferencial, el cual aborda los rasgos del liderazgo que mayor relación tienen y mejor explican la dimensión de personalidad que los agrupa. Posteriormente, se desarrolla la discusión, contrastando los hallazgos y los referentes teóricos abordados $\mathrm{y}$, finalmente, las conclusiones.

\section{Marco teórico}

El desarrollo de este trabajo se enmarca en los referentes teóricos relacionados con la conceptualización del liderazgo y las teorías asociadas, el liderazgo en las OSFL —que 
permite entender el ejercicio del liderazgo en este tipo de organizaciones- y el género, referido no a la diferenciación biológica entre los sexos - hombre-mujer-, sino a la perspectiva que permite reconocer el contexto cultural en el que hombres y mujeres interactúan.

\subsection{Liderazgo}

\subsubsection{Conceptualización de liderazgo.}

Existen varias definiciones de liderazgo que reflejan su complejidad, en la medida en que cada uno de los teóricos del tema se apoya en paradigmas diferentes a partir de los cuales basan su análisis. Son tres los elementos comunes hallados en las definiciones: el logro de las metas, la motivación de los empleados para contribuir al logro de dichas metas y el liderazgo como una capacidad para influir en otros.

En relación con el logro de las metas, se tiene que el líder interactúa con sus colaboradores para que, por medio de un esfuerzo mancomunado, sea posible alcanzar los objetivos definidos (Bennis, 1977; Zaleznik, 1990; Lussier y Achua, 2005).

Por su parte, Burns (1978), Bass (1990) y House (1992), así como Gibson, Ivancevich y Donnelly (2001), destacan la motivación como ejercicio fundamental que debe realizar el líder para hacer que sus colaboradores se comprometan con la consecución de los objetivos.

De igual forma, Katz y Kahn (1978), Hall (1983), Robbins y Coulter (2005), Dubrin (2008) y Cohen (2011), entre otros, manifiestan la necesidad de que el líder tenga la capacidad de influir en el comportamiento de los integrantes de la organización a partir de sus actitudes y valores, en el propósito de lograr los objetivos.

Así pues, analizar estos enfoques teniendo en cuenta las organizaciones objeto de estudio, o sea las fundaciones, la capacidad de influencia del líder, no solo en sus empleados, sino también en personas externas a la organización, resulta determinante para recibir las aportaciones requeridas con miras a la consecución de los objetivos y de la sostenibilidad 
organizacional. A continuación, se presentan las teorías del liderazgo a partir de los enfoques de los rasgos del líder, centrando el análisis en el inventario revisado de personalidad NEO PI-R, propuesto por Costa y McCrae (1992), cuya adaptación fue el instrumento de recolección de la información para esta investigación.

\subsubsection{Teorías del liderazgo y el liderazgo en las OSFL.}

Al analizar las características de las OSFL se evidencia que el liderazgo ejercido por quienes las dirigen debe caracterizarse por su esfuerzo en lograr la legitimidad de sus organizaciones, a través del establecimiento de unas normas claras de funcionamiento y un comportamiento ético; pero, además, por desarrollar una cultura fuerte y un compromiso con los valores organizacionales que permitan no solo lograr los objetivos, sino también los aportes de los diferentes grupos de interés.

De allí la necesidad de presentar una breve reseña de los desarrollos del liderazgo a partir de la identificación de los rasgos del líder, de los comportamientos y de las situaciones que afectan el ejercicio del liderazgo, a fin de entender esta habilidad que todo directivo debe apropiar.

La teoría de rasgos se deriva de los trabajos de Thomas Carlyle (1904), quien partió de la idea del gran hombre, con referencia a personajes de la historia universal, líderes de grandes hombres, que sirvieron como modelo. Posteriores investigaciones como, por ejemplo, la realizada por Stogdill y Bass (1990), establecieron que los factores asociados con el liderazgo probablemente podrían clasificarse en seis componentes: capacidad, logro, responsabilidad, participación, estatus y situación. De este modo, llegan a la conclusión de que los rasgos, en sí mismos, no eran suficientes para lograr un liderazgo eficaz, pues era necesario que estos fueran concordantes con las características, las actividades y las metas de sus seguidores.

Al respecto, Cowley (1931) plantea que el estudio del liderazgo es un constructo complejo integrado por una diversidad de rasgos que implica tener en cuenta tres distinciones. La primera es la diferencia entre jefe y líder, al ser el jefe quien ostenta un cargo en la jerarquía organizacional y el líder quien tiene un motivo o un programa que moviliza a los demás. La 
segunda consiste en el análisis de los rasgos a partir del individuo o de la situación; y la tercera es la distinción entre el liderazgo natural, es decir, aquel que se considera nato y el liderazgo que surge en situaciones específicas.

Dentro de los enfoques de rasgos surge un planteamiento relevante como es el esbozado por D’Alessio (2010), denominado «el modelo de los cinco factores de la personalidad», inicialmente llamado «inventario revisado de personalidad NEO PI-R», desarrollado por Costa y McCrae (1992); este es uno de los instrumentos más conocidos utilizado para medir la personalidad y es el que se utiliza en esta investigación. El instrumento analiza cinco dimensiones o dominios de la personalidad, los cuales se dividen en seis facetas, lo que permite una evaluación exhaustiva de la personalidad adulta y que se presentan en el apartado metodología.

A medida que pasó el tiempo, la teoría de rasgos dio paso a las teorías centradas en los comportamientos del líder, las cuales surgieron en la década de los cincuenta y se concentran en los comportamientos que desempeña el líder en el trabajo, es decir, en identificar acciones concretas de los líderes, tratando de explicar los estilos distintivos de los líderes eficaces o de definir la naturaleza de su labor (Lussier y Achua, 2005).

Entre las teorías del comportamiento más representativas se encuentran los estudios de la Universidad de Iowa, conducidos por Lewin, Lippitt y White (1939), quienes exploraron tres estilos de liderazgo: autocrático, democrático y laissez-faire. Otras de las investigaciones a destacar son los estudios de la Universidad de Michigan y de la Universidad del Estado de Ohio, en los que se presenta la existencia de dos tipos principales de conducta del líder: una de estructura o centrada en el trabajo, orientada a las tareas, y una de consideración o centrada en los empleados.

Con respecto a la segunda, cabe resaltar el análisis realizado por Schriesheim y Stogdill (1975), quienes compararon la estructura factorial y la complejidad de las tres versiones del instrumento que permiten analizar dichas conductas en el líder, como lo son el cuestionario de descripción del comportamiento de supervisión SBDQ (Fleishman, 1972), el cuestionario 
de descripción de la conducta del líder LBDQ (Halpin, 1957) y el formulario XII revisado del cuestionario de descripción de la conducta del líder LBDQ-XII (Stogdill, 1963).

Los investigadores encontraron que la escala SBDQ contiene ítems que son ajenos a la medición de las conductas de consideración y estructura, con énfasis en los comportamientos del líder que son punitivos y arbitrarios, mientras que el LBDQ y el LBDQ-XII se centran en la medición de los comportamientos de estructura — producción-y son más adecuados para evaluar el comportamiento del líder; especialmente el LBDQ-XII por una menor complejidad de su estructura factorial y su exitosa validación experimental.

Otra teoría del comportamiento es la parrilla gerencial o malla administrativa expuesta por Robert Blake y Jane Mouton (1967), en la que presentan cinco estilos de gerencia: gerencia 1,1, gerencia 9,1, gerencia 1,9, gerencia 5,5 y gerencia 9,9. Posteriormente, surgen los enfoques situacionales del liderazgo, entre los que se destacan la teoría situacional o por contingencia, cuyo pionero fue Fred Fiedler, quien en 1965 planteó que la eficacia de un líder se basa en el estilo del líder y su control de la situación (Fiedler, 1978, como se cita en Fiedler, Ayman y Chemers, 1995).

Otro de los enfoques es el continuo del liderazgo de Robert Tannenbaum y Warren Schmidt (1973), quienes plantean un modelo que es un continuo o un rango de conducta de liderazgo disponible para los gerentes cuando toman decisiones, el cual se desplaza desde un estilo autocrático hacia uno más democrático. Adicionalmente, el modelo de liderazgo del ciclo de vida de Paul Hersey, Ken Blanchard y Walter Natemeyer (1979) propone que el estilo de liderazgo que tiene la mayor probabilidad de éxito está determinado por la madurez del seguidor y el comportamiento del líder.

A su vez, algunos enfoques emergentes tienen en cuenta una serie de variables que afectan el ejercicio del liderazgo y se ajustan a la dinámica de las OSFL, como, por ejemplo, el liderazgo transformacional, propuesto por Bernard Bass (1990), quien plantea que este ocurre cuando los líderes amplían y elevan los intereses de sus empleados, generan conciencia y aceptación de los propósitos y la misión del grupo; además motivan a sus empleados a mirar más allá de 
su propio interés por el bien del grupo. El autor, con base en una serie de encuestas, pruebas clínicas y estudio de casos, llega a la conclusión de que los líderes transformacionales tienen las siguientes características: carisma o influencia idealizada, liderazgo inspiracional o motivación inspiracional, estimulación intelectual y consideración individualizada.

Por otra parte, el liderazgo de servicio, propuesto por Robert K. Greenleaf (1995), partió de la reflexión que el autor hace sobre la situación de la sociedad, caracterizada por la desigualdad, la corrupción y la injusticia, por lo que se requiere del surgimiento de líderes, quienes, principalmente, sean servidores; o sea, personas capaces de buscar, escuchar y a la expectativa de generar cambios positivos para la época actual.

Finalmente, algunos estudios empíricos que dan cuenta del ejercicio del liderazgo en las OSFL destacan que los elementos de liderazgo predominantes en este tipo de organizaciones son el respeto, la confianza, la honestidad y el entusiasmo; se alienta la innovación, la creatividad y hay preocupación por el bienestar de las personas; además, se resalta el perfil del líder como alguien que tiene capacidad de ejercer control social, diálogo y escucha (Espinosa, Contreras, Uribe y Barbosa, 2014; Erazo-Caicedo, Jiménez-Ruiz y López-Morales, 2014; Mejía-Londoño, Zea-Jaramillo y Pérez-Ortega, 2004; Saravia-Vergara, 2014).

A partir de lo anterior, se evidencia, en primera instancia, que las diversas teorías y los estudios empíricos acerca del liderazgo son aplicables tanto a los hombres como a las mujeres, a quienes en algún momento les corresponde ejercer su rol como líderes; en segundo lugar, que los líderes de las OSFL deben contar con los rasgos y valores referenciados, pues deben coordinar, motivar y dirigir equipos de trabajo, o sea, adquirir esas habilidades de relaciones interpersonales para lograr que la organización sea flexible con miras a lograr su sostenibilidad.

A continuación, se aborda la temática de género con el objetivo de indagar acerca de las diferentes perspectivas que han dinamizado la reflexión en torno a la concepción del rol del hombre y de la mujer en la sociedad. 


\subsubsection{Género.}

Antes de mencionar el significado de género es interesante hacer alusión a la perspectiva de género que, como lo plantea Lamas (1996), es importante en la medida en que permite explicar el porqué de la discriminación de las mujeres en la sociedad, la cual se da de manera soterrada debido a las concepciones culturales en torno a que la mujer es débil, vulnerable durante el embarazo e insustituible para cierto modelo de familia, lo que justifica su protección, aunque esta esconda una real discriminación. Estas presunciones con el tiempo se han convertido en prejuicios que llevan a que las mujeres enfrenten realidades tales como salarios más bajos y pocas posibilidades de promoción.

Así, la perspectiva de género permite reconocer el contexto cultural en el que hombres y mujeres interactúan, y del cual se derivan algunas realidades relacionadas con el reconocimiento de que existen diferencias biológicas entre hombres y mujeres, las cuales no determinan que hayan comportamientos exclusivos de uno u otro sexo; con los trabajos que deben realizar las mujeres y no los hombres, como, por ejemplo, las labores de protección y cuidado, que se consideran naturales y son asociadas culturalmente a las mujeres; y con la situación de inequidad de las mujeres en las organizaciones, evidenciada en la ocupación de pocos puestos de alta gerencia, la discriminación salarial, el hostigamiento sexual y la percepción de ineptitud en el desempeño de algunos cargos, reforzada por las actitudes de las mismas mujeres.

A partir de ese reconocimiento, la perspectiva de género «diseña acciones para garantizar la inserción de las mujeres en el mundo del trabajo y para promover su desarrollo profesional y político» (Lamas, 1996, p. 8); además, beneficia a la sociedad, al eliminar obstáculos y discriminaciones con el fin de establecer condiciones más equitativas de participación.

La definición de género y las perspectivas resultantes han sido el motivo de múltiples interpretaciones y corrientes que buscan explicar la relación entre los sexos de la especie humana. De esta manera, surgen posturas individualistas, a partir de las cuales el género se considera como atributo de un individuo que permite clasificarlo según el sexo, la identidad 
sexual o el rol social, así como posturas holistas que dimensionan el género como un ordenador social que plantea cómo la sociedad está constituida por individuos, pero también por sus normas, valores y representaciones colectivas.

Este es el caso de Rubin (1986), quien considera que el género es una construcción social; de Chodorow (1978), quien plantea que el género es un sistema de estatus; de Butler (1998), quien define el género como una construcción cultural; de Scott (2003), que lo considera una forma primaria de relaciones significantes de poder; y de Kergoat (2002), quien propone que el género es el resultado de la división del trabajo.

Bajo cualquiera de las perspectivas es necesario aclarar varios aspectos: primero, el sexo no es lo mismo que el género, pues el primero se refiere a lo biológico y el segundo a lo cultural (Lamas, 2000); segundo, la perspectiva de género ha sido útil para explicar el porqué de la discriminación de las mujeres en la sociedad (Lamas, 1996); y tercero, el análisis de género o de los sistemas sexo/género debe abordarse desde las dimensiones biológicas, sociales y psíquicas (Lamas, 2008).

Por otra parte, es pertinente analizar la subrepresentación de la mujer en los roles del liderazgo, ya que, generalmente, se asocia el éxito en la gerencia con características masculinas, relación abordada por Schein, Mueller, Lituchy y Liu (1996), quienes al analizar dicho fenómeno en estudiantes de administración de Japón y China encontraron que ambos grupos perciben que los gerentes intermedios exitosos poseen características, actitudes y temperamentos atribuidos más comúnmente a los hombres que a las mujeres.

Esto es consecuente con los resultados de estudios previos realizados en Estados Unidos, Gran Bretaña y Alemania, lo que les permite a los autores concluir que el fenómeno «pensar en el gerente, pensar en el hombre» se constituye en un fenómeno global que ha implicado el sesgo contra las mujeres en las decisiones de selección, ubicación, promoción y capacitación de la gerencia. Sin embargo, esta hipótesis de tipificación del sexo de los directivos desde la percepción de las mujeres varió entre países, debido a la diferencia de oportunidades de participación gerencial, especialmente en Estados Unidos. Finalmente, los autores llaman la 
atención sobre la necesidad de realizar un mayor número de estudios empíricos en países africanos y de América del Sur con el fin de aumentar los esfuerzos dirigidos a promover la igualdad de género en la gestión en todo el mundo.

Esta reseña da cuenta de la importancia de las posturas holistas, especialmente las planteadas por Rubin (1986) y Butler (1998), con el fin de comprender el papel que desempeñan las mujeres participantes en esta investigación, quienes ejercen la ética del cuidado en su ámbito familiar y laboral.

Por lo anterior, y en consideración a que en las bases de datos consultadas no se encontraron suficientes hallazgos empíricos que den cuenta del análisis de los rasgos del liderazgo en una población particular como lo son las mujeres directoras de fundaciones en el Valle del Cauca, surge el interés por determinar cuáles de estos rasgos son predominantes en ellas y su impacto en la dimensión de la personalidad que los integra, lo cual predice su potencial de liderazgo; en este propósito se plantean las siguientes hipótesis de investigación:

- Ho1: ninguno de los rasgos del liderazgo —ansiedad, depresión, ansiedad social, impulsividad, vulnerabilidad ante el estrés - aporta a la variación de la dimensión neurotismo.

- H1: los rasgos del liderazgo —ansiedad, depresión, ansiedad social, impulsividad, vulnerabilidad ante el estrés - aportan a la variación de la dimensión neurotismo.

- Ho2: ninguno de los rasgos del liderazgo — calidez, gregarismo, asertividad, actividad, emociones positivas - aporta a la variación de la dimensión extroversión.

- H2: los rasgos del liderazgo — calidez, gregarismo, asertividad, actividad, emociones positivas - aportan a la variación de la dimensión extroversión.

- Ho3: ninguno de los rasgos del liderazgo —-fantasía, sentimiento, acciones, ideas, valoresaporta a la variación de la dimensión apertura a nuevas experiencias. 
- H3: los rasgos del liderazgo - fantasía, sentimiento, acciones, ideas, valores - aportan a la variación de la dimensión apertura a nuevas experiencias.

- Ho4: ninguno de los rasgos del liderazgo - confianza, franqueza, altruismo, conformidad, modestia - aporta a la variación de la dimensión agradabilidad.

- H4: los rasgos del liderazgo — confianza, franqueza, altruismo, conformidad, modestiaaportan a la variación de la dimensión agradabilidad.

- Ho5: ninguno de los rasgos del liderazgo - competencia, sentido del deber, necesidad de logro, autodisciplina, deliberación — aporta a la variación de la dimensión escrupulosidad.

- H5: los rasgos del liderazgo - competencia, sentido del deber, necesidad de logro, autodisciplina, deliberación — aportan a la variación de la dimensión escrupulosidad.

\section{Metodología}

El diseño metodológico de este estudio utiliza un enfoque cuantitativo haciendo uso de los procedimientos de la investigación descriptiva, la cual permite abordar las especificidades de las organizaciones participantes, definir los rasgos del liderazgo y comprender el fenómeno estudiado, es decir, conocer cuáles de esos rasgos identificados poseen las directoras participantes y en qué grado. También se realizaron análisis inferenciales para establecer cuáles de los rasgos abordados explican y tienen el mayor impacto en el comportamiento de la dimensión o el dominio de la personalidad que los integra, lo cual se justifica en la medida en que dichas características predicen el potencial de liderazgo (Bass, 1990; Contreras, 2008).

Las OSFL objeto de estudio son fundaciones localizadas en el Valle del Cauca, creadas por personas naturales $y$, aunque es el departamento a nivel nacional con mayor número de organizaciones de este tipo (Vivas-Cortés, González-Tobito y Gómez-Sarmiento, 2015), muchas no se encuentran activas o desarrollan muy pocas actividades, no es posible localizar 
a su representante legal por desactualización de los datos de contacto o hay reticencia a participar en este tipo de estudios, pues se considera que los beneficios no son tangibles.

En razón a lo anterior, fue necesario acudir a los contactos previos obtenidos gracias al desarrollo de proyectos de intervención realizados por estudiantes universitarios en algunas de estas fundaciones dirigidas por mujeres. De allí que se trate de un muestreo por conveniencia que consistió en la invitación a participar del proyecto de investigación, del cual resultó este artículo, a 42 fundaciones, con lo que se logró la vinculación de 31 de ellas a través de una prueba piloto inicial en la que se encuestaron a 16 directoras; posteriormente, se aplicó el instrumento a 15 más.

Las fundaciones están ubicadas en Cali, Palmira, Yumbo, Jamundí y El Cerrito, y se dedican a ayudar a las poblaciones vulnerables de la región, específicamente a madres cabeza de hogar, jóvenes adolescentes en embarazo, mujeres afectadas por implantes mamarios y cáncer de seno, niños y jóvenes en situación de pobreza, violencia y abandono, niños con discapacidad, cáncer y VIH, adultos mayores y animales. Cabe destacar que los nombres de las fundaciones no se publican por motivos de confidencialidad.

El trabajo de campo consistió en la aplicación de una encuesta vía telefónica a las directoras de las fundaciones que se diligenció en copia dura. El instrumento utilizado corresponde a una elaboración propia a partir del inventario revisado de personalidad NEO PI-R, desarrollado por Paul Costa y Robert McCrae en 1992 (D'Alessio, 2010), cuyo alfa de Cronbach reportó un 0,803 , lo que indica su fiabilidad.

Esta encuesta contiene un primer apartado con preguntas sobre información sociodemográfica de las directoras tales como la edad, el nivel salarial y el nivel de formación, y un segundo bloque referente a los rasgos del liderazgo, en el que se utilizó la escala diferencial semántica, a partir de la cual las mujeres encuestadas respondían entre 0 y 5 , dependiendo de cómo se consideraban ellas, al explicarles la característica de las personas que obtenían una puntuación baja y alta en cada rasgo (véase la Tabla 1). 
Tabla 1. Significado de la escala utilizada en la encuesta-rasgos del liderazgo

\begin{tabular}{|c|c|c|c|c|c|c|}
\hline \multirow{3}{*}{ Dimensión } & \multicolumn{6}{|c|}{ Significado escala } \\
\hline & $\begin{array}{c}0 \\
\text { No tiene el } \\
\text { rasgo }\end{array}$ & $\begin{array}{c}1 \\
\text { Nada }\end{array}$ & $\begin{array}{c}2 \\
\text { Poco }\end{array}$ & $\begin{array}{c}3 \\
\text { Nivel } \\
\text { medio }\end{array}$ & $\begin{array}{c}4 \\
\text { Tiene el rasgo }\end{array}$ & $\begin{array}{c}5 \\
\text { Muy }\end{array}$ \\
\hline & \multicolumn{6}{|c|}{ Rasgo } \\
\hline Neurotismo & & $\begin{array}{c}\mathrm{N} 1 \\
\text { Ansiedad }\end{array}$ & $\begin{array}{c}\text { N2 } \\
\text { Depresión }\end{array}$ & $\begin{array}{l}\mathrm{N} 3 \\
\text { Ansiedad } \\
\text { social }\end{array}$ & $\begin{array}{c}\mathrm{N} 4 \\
\text { Impulsividad }\end{array}$ & $\begin{array}{c}\mathrm{N} 5 \\
\text { Vulnerabilidad } \\
\text { ante el estrés }\end{array}$ \\
\hline Interpretación & & Ansiosa & Depresiva & $\begin{array}{c}\text { Ansiosa } \\
\text { socialmente }\end{array}$ & $\begin{array}{l}\text { Tolerante a la } \\
\text { frustración }\end{array}$ & Vulnerable \\
\hline Extroversión & & $\begin{array}{c}\text { E1 } \\
\text { Calidez }\end{array}$ & $\begin{array}{c}\text { E2 } \\
\text { Gregarismo }\end{array}$ & $\begin{array}{c}\text { E3 } \\
\text { Asertividad }\end{array}$ & $\begin{array}{c}\text { E4 } \\
\text { Actividad }\end{array}$ & $\begin{array}{c}\text { E5 } \\
\text { Emociones } \\
\text { positivas }\end{array}$ \\
\hline Interpretación & & Cálida & Gregaria & Asertiva & Activa & Alegre \\
\hline $\begin{array}{l}\text { Apertura } \\
\text { nuevas } \\
\text { experiencias }\end{array}$ & & $\begin{array}{c}01 \\
\text { Fantasía }\end{array}$ & $\begin{array}{c}02 \\
\text { Sentimiento }\end{array}$ & $\begin{array}{c}03 \\
\text { Acciones }\end{array}$ & $\begin{array}{c}04 \\
\text { Ideas }\end{array}$ & $\begin{array}{c}05 \\
\text { Valores }\end{array}$ \\
\hline Interpretación & & Fantasiosa & Sentimental & Flexible & $\begin{array}{c}\text { Curiosa } \\
\text { intelectualmente }\end{array}$ & Normativa \\
\hline Agradabilidad & & $\begin{array}{c}\text { A1 } \\
\text { Confianza }\end{array}$ & $\begin{array}{c}\text { A2 } \\
\text { Franqueza }\end{array}$ & $\begin{array}{c}\text { A3 } \\
\text { Altruismo }\end{array}$ & $\begin{array}{c}\text { A4 } \\
\text { Conformidad }\end{array}$ & $\begin{array}{c}\text { A5 } \\
\text { Modestia }\end{array}$ \\
\hline Interpretación & & Confiada & Franca & Altruista & Agresiva & Modesta \\
\hline Escrupulosidad & & $\begin{array}{c}\mathrm{C} 1 \\
\text { Competencia }\end{array}$ & $\begin{array}{l}\text { C2 Sentido } \\
\text { del deber }\end{array}$ & $\begin{array}{c}\text { C3 } \\
\text { Necesidad } \\
\text { de logro }\end{array}$ & $\begin{array}{c}\text { C4 } \\
\text { Autodisciplina }\end{array}$ & $\begin{array}{c}\text { C5 } \\
\text { Deliberación }\end{array}$ \\
\hline Interpretación & & Competente & Responsable & Ambiciosa & Autodisciplinada & $\begin{array}{c}\text { Cautelosa y } \\
\text { analítica }\end{array}$ \\
\hline
\end{tabular}

Fuente. Elaboración propia.

Una vez obtenida la información se procedió a realizar el análisis descriptivo mediante el software Excel y SPSS, lo que permitió hallar el alfa de Cronbach y las frecuencias según las opciones de respuestas dadas, con el fin de establecer la información sociodemográfica y los rasgos del liderazgo predominantes en las directoras de las fundaciones objeto de estudio.

Seguidamente, se efectuó el análisis inferencial que consistió, en primer lugar, en el cálculo de los estadísticos descriptivos con el fin de determinar a través del coeficiente de variación cuáles de los rasgos del liderazgo afectan a los demás en cada una de las dimensiones de la personalidad. Cabe resaltar que los cálculos que a continuación se detallan se realizaron para cada una de las cinco dimensiones de la personalidad y sus correspondientes rasgos del liderazgo. 
En segundo lugar, se calculó el coeficiente de correlación de Pearson ${ }^{3}$ con el fin de identificar aquellos rasgos que tienen relación directa con la dimensión de la personalidad que los agrupa, al ser aquellos que reportan un nivel de significancia menor a 0,05 -95\% de confianza en que la correlación es verdadera y 5 \% de probabilidad de error-.

Así, a mayor valor del coeficiente, mayor es la correlación entre las variables, que puede ser positiva, indicando que la proporcionalidad es directa; o sea, mientras la directora exhiba conductas asociadas a un rasgo determinado, mayor será su nivel en la dimensión analizada. Entonces, puede ser cero, significando que no existe correlación alguna entre las variables; o negativo, es decir, que la proporcionalidad es indirecta, es decir, cuando la directora exhibe más comportamientos de un rasgo determinado, menor es su nivel en la dimensión correspondiente.

En tercera instancia, se procede a calcular el coeficiente de determinación $-\mathrm{R}$ cuadradopara establecer el porcentaje de la variación de la dimensión de la personalidad — variable dependiente-, debido a la variación de los rasgos del liderazgo que la integran — variables independientes-.

En cuarto lugar, se realiza el cálculo del análisis de varianza factorial —Anova de varios factores- con el fin de «evaluar el efecto de dos o más variables independientes sobre una variable dependiente» (Hernández, Fernández y Baptista, 2010, p. 326); este permite probar las hipótesis planteadas en torno a si los rasgos del liderazgo en su conjunto aportan o no a la variación de la dimensión de la personalidad que los integra, concluyendo que sí aportan cuando el nivel de significancia es menor a 0,05.

Luego, se procede a analizar cuáles de los rasgos del liderazgo en forma individual asociados a cada dimensión aportan a su variación, a través del cálculo de la ecuación de regresión y sus coeficientes — coeficiente no estandarizado $\mathrm{B}^{4}-$, que permiten aceptar o negar las

\footnotetext{
${ }^{3}$ Es una estadística apropiada para variables medidas por intervalos o razón y para relaciones lineales. Su valor puede variar de 1 a +1 (Hernández et al., 2010, p. 331).

${ }^{4}$ En regresión múltiple, los coeficientes de regresión estandarizados permiten valorar la importancia relativa de cada variable independiente dentro de la ecuación (Pardo y Ruiz, 2005, p. 344).
} 
hipótesis esbozadas en relación con si cada rasgo aporta a la explicación de la variación de la dimensión correspondiente. La aceptación ocurre cuando el nivel de significancia es menor a 0,05 .

Finalmente, se realiza la estimación del modelo con los rasgos del liderazgo que aportan a dicha variación, a partir del cálculo del R cuadrado, el Anova y la ecuación de regresión para concluir que, entre mayores sean los valores asociados a cada rasgo del liderazgo, si permanecen constantes los demás, el nivel de la dimensión analizada tenderá a incrementarse en las directoras participantes.

\section{Resultados}

En este apartado se presentan los resultados obtenidos a partir del análisis descriptivo con relación al perfil de las mujeres participantes y de las fundaciones que dirigen, y el nivel que poseen en cada rasgo del liderazgo en cada una de las dimensiones de la personalidad estudiadas. El análisis inferencial, por su parte, muestra los rasgos del liderazgo que tienen mayor relación con la dimensión de la personalidad correspondiente y mejor explican su variación.

\subsection{Perfil de las directoras participantes y sus fundaciones}

De acuerdo con los resultados obtenidos, se evidencia que el $25,8 \%$ de las fundaciones ubicadas en las ciudades de Cali, Palmira, Yumbo, Jamundí y El Cerrito atienden a niños y jóvenes en situación de pobreza, violencia y abandono; otro 25,8 \% a los adultos mayores y un $19,4 \%$ a los animales abandonados.

Al abordar la edad de las directoras se tiene que un 32,3 \% están entre los 41 y 50 años, y un $29 \%$ entre los 51 y 60 años. Respecto del nivel salarial, se encuentra que el 35,5 \% de ellas no tiene un salario establecido y un 32,3\% gana entre COP 1501000 y 2000000 de pesos colombianos. 
Respecto al nivel de formación de las directoras, se encuentra que el 48,4 \% de ellas tiene educación superior profesional, el 16,1 \% posee educación media completa y solo el 6,5 \% tiene título de maestría.

\subsection{Análisis de las dimensiones del liderazgo: neurotismo, extroversión,} apertura a nuevas experiencias, agradabilidad y escrupulosidad, y los rasgos asociados a cada dimensión

A continuación, se presentan los resultados obtenidos a partir de la percepción de las directoras en cuanto a los rasgos de su liderazgo, juzgando qué tanto coincidían con ellas las características de cada rasgo cuando se tiene una puntuación baja y alta; se presenta primero la dimensión y luego los rasgos asociados.

Al abordar las dimensiones de la personalidad, específicamente el neurotismo, se encuentra que el $51,7 \%$ de las directoras son estables y muy estables emocionalmente, y el 41,9\% se encuentran en un nivel medio (véase la Tabla 2). En los rasgos que abarcan esta dimensión, la mayoría de las participantes son poco o nada ansiosas, lo cual indica que no son temerosas y, en cambio, son relajadas y calmadas (D'Alessio, 2010); no son depresivas, es decir, no se desalientan con facilidad (Costa y McCrae, 1992); se perciben entre poco y medio ansiosas socialmente, o sea que pueden experimentar sentimientos de inferioridad y perturbarse frente a ciertas situaciones sociales (Costa y McCrae, 1992); son poco impulsivas, lo cual significa que son tolerantes a la frustración y controlan sus emociones; y son poco o nada vulnerables ante el estrés, lo que demuestra su capacidad de enfrentar situaciones difíciles (D’Alessio, 2010).

Respecto a la extroversión se encuentra que, según la autopercepción de las directoras, estas se consideran, en su mayoría, extrovertidas y muy extrovertidas (véase la Tabla 2), evidenciado en sus niveles altos de calidez, al ser afectivas y amigables (Costa y McCrae, 1992); se destaca el gregarismo, lo cual significa que les agrada tener compañía y disfrutan del contacto social (D’Alessio, 2010); al igual que la asertividad, indicando que son fuertes, dominantes y líderes de grupo, lo que coincide con la actividad que realizan; también la 
actividad, o sea que son vigorosas y necesitan mantenerse ocupadas (Costa y McCrae, 1992); y emociones positivas, significando que experimentan emociones como alegría, felicidad y amor (D’Alessio, 2010).

En relación con la apertura a nuevas experiencias, se establece que las directoras consideran que están entre un nivel medio y alto (véase la Tabla 2), lo cual es evidente en que tienden a ser poco fantasiosas, es decir, son personas que no divagan $\mathrm{y}$, por el contrario, se centran en aspectos concretos (D’Alessio, 2010). Así mismo, son sentimentales, lo cual significa que, en su mayoría, tienen una experiencia profunda en relación con la intensidad de sus emociones (Costa y McCrae, 1992). También se perciben como muy flexibles —rasgo acciones-, es decir, son más proclives a la variedad que a la rutina, lo que facilita la adaptación a los cambios; muy curiosas intelectualmente —rasgo ideas—, indicando que les gusta aprender cosas nuevas; y poco o nada normativas, o sea que se inclinan a reexaminar valores sociales, políticos y religiosos (Costa y McCrae, 1992).

Al analizar la agradabilidad se encuentra que las participantes se ubican en un nivel alto y muy alto en esta dimensión (véase la Tabla 2), lo cual se refleja en que son confiadas, pues creen en las buenas intenciones de los demás, aunque en ocasiones pueden ser escépticas (D'Alessio, 2010). Igualmente, se perciben como personas francas al ser sinceras y honestas, se ubican entre un nivel medio y muy alto en el altruismo, evidenciándose así su preocupación por otros, lo cual coincide con su rol cuidador, pues muchas de ellas crearon sus fundaciones para ayudar a los más vulnerables. Finalmente, en cuanto al rasgo conformidad se encuentra que la mayoría se percibe como poco o nada agresivas, lo que demuestra su inclinación por defender a los demás, rechazar la agresión y perdonar (Costa y McCrae, 1992).

Al abordar la dimensión escrupulosidad se observa que la mayoría de las directoras son muy escrupulosas, o sea, orientadas al logro (véase la Tabla 2). Esto se concluye porque obtuvieron niveles altos en los rasgos: competencia, pues se consideran personas capaces, creen en sus habilidades y se sienten preparadas para enfrentar la vida; sentido del deber, dado que son responsables, pues obran con rectitud, apegadas a principios éticos y a sus 
obligaciones morales (D’Alessio, 2010); necesidad de logro, dado que se perciben como ambiciosas, persistentes y que trabajan duro por alcanzar sus metas (Zopiatis y Constanti, 2012); autodisciplina, lo que significa que no se desaniman fácilmente y tienen la habilidad de automotivarse para terminar un trabajo (Costa y McCrae, 1992); y deliberación, lo cual indica que son cautelosas y analíticas, es decir, que piensan antes de actuar, considerando las consecuencias de sus decisiones (D’Alessio, 2010).

Tabla 2. Dimensiones de la personalidad: neurotismo, extroversión, apertura a nuevas experiencias, agradabilidad y escrupulosidad

\begin{tabular}{|c|c|c|c|c|c|}
\hline Neurotismo/descripción & Frecuencia & $\%$ & Extroversión/descripción & Frecuencia & $\%$ \\
\hline Muy estable emocionalmente & 2 & 6,5 & Término medio & 4 & 12,9 \\
\hline Estable emocionalmente & 14 & 45,2 & Extrovertida & 20 & 64,5 \\
\hline Término medio & 13 & 41,9 & Muy extrovertida & 7 & 22,6 \\
\hline Poco estable emocionalmente & 2 & 6,5 & & & \\
\hline Total & 31 & 100,0 & Total & 31 & 100,0 \\
\hline $\begin{array}{c}\text { Apertura a nuevas } \\
\text { experiencias/descripción }\end{array}$ & Frecuencia & $\%$ & Escrupulosidad/descripción & Frecuencia & $\%$ \\
\hline $\begin{array}{l}\text { Poco abierta a nuevas } \\
\text { experiencias }\end{array}$ & 2 & 6,5 & Término medio & 1 & 3,2 \\
\hline Término medio & 14 & 45,2 & Escrupulosa & 16 & 51,6 \\
\hline Abierta a nuevas experiencias & 15 & 48,4 & Muy escrupulosa & 14 & 45,2 \\
\hline Total & 31 & 100,0 & Total & 31 & 100,0 \\
\hline Agradabilidad/descripción & Frecuencia & $\%$ & & & \\
\hline Término medio & 2 & 6,5 & & & \\
\hline Agradable & 25 & 80,6 & & & \\
\hline Muy agradable & 4 & 12,9 & & & \\
\hline Total & 31 & 100,0 & & & \\
\hline
\end{tabular}

Fuente. Elaboración propia.

\subsection{Análisis de los rasgos del liderazgo que tienen mayor relación con las dimensiones de la personalidad y que mejor explican su variación}

En este apartado se muestran los análisis realizados para cada una de las dimensiones de la personalidad con el fin de probar las hipótesis planteadas en torno a si los rasgos del liderazgo asociados a cada dimensión aportan a su variación y, en caso afirmativo, estimar los parámetros del modelo considerando solo los rasgos que influyen en la dimensión correspondiente. 


\subsubsection{Neurotismo.}

Una vez calculados los estadísticos descriptivos, se procedió a encontrar el coeficiente de correlación de Pearson, lo cual evidencia que los rasgos del liderazgo que afectan positivamente a esta dimensión son, en su orden, la ansiedad social, reportando 0,667, la depresión con 0,501 y la vulnerabilidad antes el estrés con 0,432 (véase la Tabla 3).

Seguidamente, el cálculo del coeficiente de determinación R cuadrado reportó 0,734, lo cual quiere decir que los rasgos del liderazgo que integran esta dimensión explican un 73,4 \% del neurotismo en las directoras participantes.

Tabla 3. Coeficientes de correlación de Pearson dimensión neurotismo

\begin{tabular}{|c|c|c|c|c|c|c|c|}
\hline \multicolumn{8}{|c|}{ Correlaciones } \\
\hline & & Neurotismo & Ansiedad & Depresión & $\begin{array}{c}\text { Ansiedad } \\
\text { social }\end{array}$ & Impulsividad & $\begin{array}{l}\text { Vulnerabilidad } \\
\text { ante el estrés }\end{array}$ \\
\hline $\begin{array}{l}\text { Correlación de } \\
\text { Pearson }\end{array}$ & Neurotismo & 1,000 & 0,232 & 0,501 & 0,667 & 0,306 & 0,432 \\
\hline Sig. —unilateral- & Neurotismo & & 0,113 & 0,003 & 0,000 & 0,053 & 0,010 \\
\hline
\end{tabular}

Fuente. Elaboración propia.

Luego, se realizó el análisis de la prueba Anova, que registró un F de 12,71 y un nivel de significancia igual a 0,000 y, dado que es menor a 0,05, se rechaza la hipótesis nula Ho1 y se acepta la hipótesis alterna H1, correspondiente a que algunos de los rasgos estudiados aportan al neurotismo en las directoras.

Posteriormente, con el fin de analizar cuáles de estos rasgos aportan o no a la variación del neurotismo y así aceptar o rechazar las hipótesis correspondientes, se calculó la ecuación de regresión y el coeficiente no estandarizado B, con lo cual se obtiene el nivel de significancia para cinco rasgos que integran esta dimensión, y con aquellos que reportaron un nivel de significancia menor a 0,05 se calculó nuevamente el coeficiente de determinación, la prueba Anova y el coeficiente no estandarizado B a fin de plantear el modelo, tal como se muestra en la tabla 4. 
Tabla 4. Modelo dimensión neurotismo

\begin{tabular}{|c|c|c|c|c|c|}
\hline \multicolumn{6}{|c|}{ Coeficientes ${ }^{a}$} \\
\hline Modelo & R cuadrado & & $\begin{array}{l}\text { Coeficiente no } \\
\text { estandarizado B }\end{array}$ & $\mathbf{t}$ & Sig. \\
\hline \multirow[t]{4}{*}{1} & \multirow[t]{4}{*}{0,750} & -Constante- & 0,545 & 2,382 & 0,025 \\
\hline & & Depresión & 0,209 & 4,324 & 0,000 \\
\hline & & Ansiedad social & 0,401 & 6,874 & 0,000 \\
\hline & & $\begin{array}{l}\text { Vulnerabilidad } \\
\text { ante el estrés }\end{array}$ & 0,166 & 2,897 & 0,007 \\
\hline
\end{tabular}

Fuente. Elaboración propia.

Así, los rasgos del liderazgo que aportan a la explicación del neurotismo son la depresión, la ansiedad social y la vulnerabilidad ante el estrés, significando que entre mayores sean los valores asociados a cada uno de estos rasgos, si permanecen constantes los demás, el neurotismo en las directoras tiende a incrementarse, es decir, a ser poco estables emocionalmente; se destaca la ansiedad social, lo cual implica que entre más sentimientos de inferioridad experimenten las mujeres participantes, mayor será su nivel de neurotismo.

\subsubsection{Extroversión.}

En este apartado se encuentra que los rasgos del liderazgo que influyen positivamente en la dimensión extroversión son la calidez, el gregarismo, la actividad y las emociones positivas; se reportan coeficientes de correlación de Pearson de 0,544, 0,719, 0,423 y 0,508, respectivamente (véase la Tabla 5). Esto significa que, mientras las directoras de las fundaciones exhiban las conductas asociadas a estos rasgos, serán más extrovertidas. 
Tabla 5. Coeficientes de correlación de Pearson dimensión extroversión

\begin{tabular}{|c|c|c|c|c|c|c|c|}
\hline \multicolumn{8}{|c|}{ Correlaciones } \\
\hline & & Extroversión & Calidez & Gregarismo & Asertividad & Actividad & $\begin{array}{c}\text { Emociones } \\
\text { positivas }\end{array}$ \\
\hline $\begin{array}{l}\text { Correlación de } \\
\text { Pearson }\end{array}$ & Extroversión & 1,000 & 0,544 & 0,719 & 0,270 & 0,423 & 0,508 \\
\hline $\begin{array}{l}\text { Sig. - } \\
\text { unilateral- }\end{array}$ & Extroversión & & 0,001 & 0,000 & 0,071 & 0,009 & 0,002 \\
\hline
\end{tabular}

Fuente. Elaboración propia.

Al calcular el coeficiente de determinación se obtuvo un 0,795, lo cual indica que los rasgos del liderazgo que componen esta dimensión explican un 79,5\% de la extroversión en las mujeres líderes. Luego, la prueba Anova reportó un F de 19,347 y un nivel de significancia de 0,000 que permite aceptar la hipótesis alterna $\mathrm{H} 2$ referente a que los rasgos asociados a esta dimensión están aportando a su variación.

Así, se procedió a corroborar las hipótesis planteadas en torno a cuáles de los rasgos de esta dimensión aportan a su variación, y se encontró que son la calidez, el gregarismo y la actividad, tal como se presenta en la tabla 6; esto significa que, a mayor valor del rasgo, si permanecen constantes los demás, la extroversión en las directoras será evidente, de modo que se refleja en su afectividad, sociabilidad y energía, donde es preponderante el rasgo actividad.

Tabla 6. Modelo dimensión extroversión

\begin{tabular}{|c|c|c|c|c|c|}
\hline \multicolumn{6}{|c|}{ Coeficientes $^{a}$} \\
\hline Modelo & $\mathrm{R}$ cuadrado & & $\begin{array}{l}\text { Coeficiente no } \\
\text { estandarizado B }\end{array}$ & $\mathbf{t}$ & Sig. \\
\hline \multirow[t]{4}{*}{2} & \multirow[t]{4}{*}{0,747} & Constante- & 1,109 & 3,114 & 0,004 \\
\hline & & Calidez & 0,190 & 3,294 & 0,003 \\
\hline & & Gregarismo & 0,261 & 4,703 & 0,000 \\
\hline & & Actividad & 0,306 & 5,217 & 0,000 \\
\hline \multicolumn{6}{|c|}{ a. Variable dependiente: EXTROVERSIÓN } \\
\hline Extrover & $109+0$ & tividad $+0,190$ & $+0,261$ Gregaris & & \\
\hline
\end{tabular}

Fuente. Elaboración propia. 


\subsubsection{Apertura a nuevas experiencias.}

Al abordar esta dimensión se evidenció, al calcular el coeficiente de correlación de Pearson, que todos los rasgos que la integran - fantasía, sentimiento, acciones y valores - influyen positivamente en esta apertura, excepto las ideas (véase la Tabla 7); esto quiere decir que si las directoras muestran conductas asociadas a estas características del liderazgo tendrán mayor apertura a nuevas experiencias, o sea, a conocer y experimentar. Los rasgos que mayor influencia tienen en esta apertura son las acciones, con un coeficiente de Pearson de 0,795, lo que indica la tendencia de las líderes a preferir la novedad y los valores, que registró un coeficiente de 0,649 , significando su disposición a reexaminar los valores propios y aquellos que son impuestos.

Por otra parte, el coeficiente de determinación, o sea el R cuadrado, permitió establecer que los rasgos asociados a esta dimensión explican un 88,6\% de la apertura a nuevas experiencias en las directoras.

Tabla 7. Coeficientes de correlación de Pearson dimensión apertura a nuevas experiencias

\begin{tabular}{|c|c|c|c|c|c|c|c|}
\hline \multicolumn{8}{|c|}{ Correlaciones } \\
\hline & & Apertura & Fantasía & Sentimiento & Acciones & Ideas & Valores \\
\hline Correlación de Pearson & Apertura & 1,000 & 0,424 & 0,380 & 0,795 & 0,243 & 0,649 \\
\hline Sig. - unilateral- & Apertura & & 0,009 & 0,018 & 0,000 & 0,094 & 0,000 \\
\hline
\end{tabular}

Fuente. Elaboración propia.

Adicionalmente, la prueba Anova que dio como resultado un F de 38,948 y un nivel de significancia igual a 0,000 permite aceptar la hipótesis H3, es decir, que los rasgos analizados aportan a la variación de la dimensión apertura a nuevas experiencias. Posteriormente, al estimar los parámetros del modelo con todos los rasgos que abarcan esta dimensión y luego con los que reportaron un nivel de significancia menor al $5 \%$, se encontró que entre más acentuadas sean las conductas asociadas a los rasgos sentimiento, acciones, ideas y valores de las directoras, serán más abiertas a nuevas experiencias; así, los dos primeros son los que más influyen en esta dimensión de la personalidad (véase la Tabla 8). 


\subsubsection{Agradabilidad.}

El abordaje de esta dimensión permitió establecer, a partir del cálculo del coeficiente de correlación de Pearson, que los rasgos que mayor impacto positivo tienen en esta dimensión son, en su orden, la confianza $-0,608-$, la conformidad $-0,600-$, el altruismo $-0,387-$ y la franqueza - 0,341-. Esto significa que si las directoras desarrollan estos rasgos serán más agradables (véase la Tabla 9).

Tabla 8. Modelo dimensión apertura a nuevas experiencias

\begin{tabular}{|c|c|c|c|c|c|}
\hline \multicolumn{6}{|c|}{ Coeficientes $^{a}$} \\
\hline Modelo & R cuadrado & & $\begin{array}{c}\text { Coeficiente no } \\
\text { estandarizado B }\end{array}$ & $\mathbf{t}$ & Sig. \\
\hline \multirow[t]{5}{*}{3} & \multirow[t]{5}{*}{0,850} & -Constante- & 0,046 & 0,141 & 0,889 \\
\hline & & Sentimiento & 0,219 & 4,841 & 0,000 \\
\hline & & Acciones & 0,300 & 6,682 & 0,000 \\
\hline & & Ideas & 0,204 & 3,512 & 0,002 \\
\hline & & Valores & 0,193 & 3,446 & 0,002 \\
\hline
\end{tabular}

Fuente. Elaboración propia.

Posteriormente, el coeficiente de determinación dio como resultado 0,560 , lo cual indica que los rasgos del liderazgo estudiados explican un $56 \%$ de la agradabilidad en las mujeres directoras, de modo que es el valor más bajo en comparación con las anteriores dimensiones.

Tabla 9. Coeficientes de correlación de Pearson dimensión agradabilidad

\begin{tabular}{|l|c|c|c|c|c|c|c|}
\hline \multicolumn{7}{|c|}{ Correlaciones } \\
\hline $\begin{array}{l}\text { Correlación de } \\
\text { Pearson }\end{array}$ & Agradabilidad & 1,000 & 0,608 & 0,341 & 0,387 & 0,600 & 0,070 \\
\hline $\begin{array}{l}\text { Sig. - } \\
\text { unilateral- }\end{array}$ & Agradabilidad & & 0,000 & 0,033 & 0,017 & 0,000 & 0,357 \\
\hline
\end{tabular}

Fuente. Elaboración propia.

Esta evidencia explica el hecho de que al calcular la prueba Anova, la ecuación de regresión y el coeficiente no estandarizado B, ninguno de los rasgos componentes de esta dimensión 
reportaron un nivel de significancia menor a 0,05, lo cual implica que no están aportando a la explicación de la agradabilidad en las líderes y, por tanto, se acepta la hipótesis nula Ho4 que esboza cómo ninguno de los rasgos del liderazgo — confianza, franqueza, altruismo, conformidad, modestia - aporta a la variación de la dimensión agradabilidad (véase la Tabla 10).

Tabla 10. Modelo dimensión agradabilidad

\begin{tabular}{|c|c|c|c|c|c|}
\hline \multicolumn{6}{|c|}{ Coeficientes $^{\mathbf{a}}$} \\
\hline Modelo & $R$ cuadrado & & $\begin{array}{l}\text { Coeficiente no } \\
\text { estandarizado B }\end{array}$ & $\mathbf{t}$ & Sig. \\
\hline \multirow[t]{6}{*}{4} & \multirow[t]{6}{*}{0,560} & -Constante- & 0,776 & 4,795 & 0,000 \\
\hline & & Confianza & 0,093 & 1,377 & 0,181 \\
\hline & & Franqueza & 0,159 & 1,679 & 0,106 \\
\hline & & Altruismo & 0,071 & 1,787 & 0,087 \\
\hline & & Conformidad & 0,102 & 1,729 & 0,097 \\
\hline & & Modestia & 0,026 & 0,339 & 0,737 \\
\hline \multicolumn{6}{|c|}{ a. Variable dependiente: Agradabilidad } \\
\hline \multicolumn{6}{|c|}{$\begin{array}{l}\text { Agradabilidad }=0,776+0,026 \text { Modestia }+0,102 \text { Conformidad }+0,159 \text { Franqueza }+0,071 \text { Altruismo }+0,093 \\
\text { Confianza. }\end{array}$} \\
\hline
\end{tabular}

Fuente. Elaboración propia.

\subsubsection{Escrupulosidad.}

Al abordar esta última dimensión se encontró que los rasgos que la afectan positivamente son la deliberación, reportando un coeficiente de Pearson de 0,76; seguido de la competencia -0,588- y de la autodisciplina -0,408-. Así que mientras las directoras exhiban con mayor vehemencia los comportamientos asociados a estos rasgos, se esperaría que sean más escrupulosas, o sea, logro-motivadas para alcanzar sus metas (Tabla 11). 
Tabla 11. Coeficientes de correlación de Pearson dimensión escrupulosidad

\begin{tabular}{|l|l|c|c|c|c|c|c|}
\hline \multicolumn{2}{|c|}{} & \multicolumn{9}{|c|}{ Correlaciones } \\
\hline & Escrupulosidad & Competencia & $\begin{array}{c}\text { Sentido } \\
\text { del } \\
\text { deber }\end{array}$ & $\begin{array}{c}\text { Necesidad } \\
\text { de logro }\end{array}$ & Autodisciplina & Deliberación \\
\hline $\begin{array}{l}\text { Correlación de } \\
\text { Pearson }\end{array}$ & Escrupulosidad & 1,000 & 0,588 & 0,252 & 0,250 & 0,408 & 0,760 \\
\hline $\begin{array}{l}\text { Sig. }- \\
\text { unilateral- }\end{array}$ & Escrupulosidad & & 0,000 & 0,086 & 0,088 & 0,011 & 0,000 \\
\hline
\end{tabular}

Fuente. Elaboración propia.

Por otra parte, el coeficiente de determinación reportó un valor de 0,775 , lo cual indica que los rasgos que integran esta dimensión explican un 77,5 \% de la escrupulosidad en las mujeres participantes. A su vez, la prueba Anova registró un F de 17,187 y un nivel de significancia de 0,000, lo que permite aceptar la hipótesis alterna H5, según la cual algunos de los rasgos analizados aportan a la escrupulosidad en las directoras.

Finalmente, con el fin de determinar los rasgos específicos que aportan a la variación de la escrupulosidad se halla la ecuación de regresión, dando como resultado que se trata de los rasgos competencia, sentido del deber y deliberación; es decir, entre mayores sean sus valores, la escrupulosidad en las directoras tiende a incrementarse, destacándose la deliberación, es decir, que entre más cautelosas y analíticas sean, mayor será su nivel de escrupulosidad (véase la Tabla 12).

Tabla 12. Modelo dimensión escrupulosidad

\begin{tabular}{|c|c|c|c|c|c|}
\hline \multicolumn{6}{|c|}{ Coeficientes $^{a}$} \\
\hline Modelo & $R$ cuadrado & & $\begin{array}{l}\text { Coeficiente no } \\
\text { estandarizado B }\end{array}$ & $\mathbf{t}$ & Sig. \\
\hline \multirow[t]{4}{*}{5} & \multirow[t]{4}{*}{0,725} & -Constante- & 0,672 & 1,215 & 0,235 \\
\hline & & Competencia & 0,233 & 2,232 & 0,034 \\
\hline & & Sentido del deber & 0,266 & 3,116 & 0,004 \\
\hline & & Deliberación & 0,397 & 5,390 & 0,000 \\
\hline
\end{tabular}

Fuente. Elaboración propia. 
A continuación, se presenta la discusión de los resultados que implica el contraste de los hallazgos obtenidos con investigaciones y estudios empíricos relacionados.

\section{Discusión}

En este apartado se presenta la discusión de los resultados según los objetivos de investigación propuestos y que abordan los rasgos del liderazgo preponderantes en las directoras de las fundaciones participantes y su correlación con la dimensión de la personalidad que los integra.

Las organizaciones sin fines de lucro se crean con el fin de lograr objetivos y, en ese propósito, es indispensable el liderazgo de la dirección, que se traduce en esa capacidad de influir en los diferentes grupos de interés para lograr la sostenibilidad de sus aportes y, por ende, de la organización. De allí la importancia del estudio de los rasgos de los líderes que ayudan a predecir ciertas variables organizacionales, como, por ejemplo, la satisfacción laboral, la toma de decisiones y la motivación para correr riesgos, entre otras (Malouff, Thorsteinsson y Schutte, 2006, Nicholson, Fenton-O'Creevy, Soane y Willman, 2005, Weber, Blais y Betz, 2002).

A partir de los resultados encontrados en esta investigación, se observa que las líderes participantes son estables emocionalmente, lo cual significa que tienen un nivel bajo en la dimensión neurotismo y que su capacidad para manejar el estrés y superar el fracaso les posibilita corregir los errores tomando las acciones necesarias para mantener el desempeño (Bass, 2008); esto es tarea cotidiana de un líder para asegurar en buena parte la satisfacción y el esfuerzo extra de sus colaboradores (Parra, Useche y Barbosa, 2010), especialmente en este tipo de organizaciones, en las que la mayoría de ellos son voluntarios.

Al analizar descriptivamente los rasgos del liderazgo asociados a la dimensión neurotismo, se evidencia que los más preponderantes en las directoras, según su percepción, son la poca vulnerabilidad ante el estrés, es decir, su alta capacidad para enfrentar situaciones difíciles, así como su alta tolerancia a la frustración —impulsividad-, características determinantes 
del liderazgo de las mujeres que han constituido organizaciones para favorecer a la población en condiciones de desplazamiento (Moreno, Badillo y Marín, 2006).

Sin embargo, algunas de las líderes opinan que suelen tener sentimientos de inferioridad ansiedad social-, lo cual puede explicarse por el rol femenino de cuidado y protección que culturalmente se ha arraigado en la sociedad, que en ocasiones resulta en una percepción de ineptitud en el desempeño de algunos cargos ejercidos por mujeres, reforzada por las actitudes de las mismas mujeres (Lamas, 1996), o en una evaluación menos favorable del potencial del liderazgo de las mujeres en comparación con el de los hombres (Eagly y Karau, 2002).

El estudio de la correlación entre la dimensión neurotismo y los rasgos del liderazgo que la componen permitió encontrar tanto en la prueba del coeficiente de Pearson como en el análisis de los parámetros del modelo que la ansiedad social, la depresión y la vulnerabilidad ante el estrés son las características que están relacionadas y mejor explican la variación de esta dimensión.

Estos resultados, especialmente en relación con la poca vulnerabilidad ante el estrés de las participantes, coinciden con algunas investigaciones que muestran cómo el neurotismo es el predictor más influyente de la resiliencia — capacidad de mantenerse relativamente estable a pesar de las adversidades-, de manera que es más fuerte en las mujeres que en los hombres (Limura y Taku, 2018); así mismo, del dominio del entorno - bajo nivel de ansiedad social-, y la autonomía y autoaceptación — bajo nivel de depresión- (González-Villalobos y Marrero, 2017).

Por otra parte, se observa que las líderes participantes tienen un nivel alto en la dimensión extroversión. Esto indica que son bastante sociables y gregarias, que pueden usar su capacidad para estimular la interacción social (Do y Minbashian, 2014), lo cual posibilita que sus seguidores se identifiquen con ellas, resultando en un comportamiento laboral productivo (Akinwale, 2019). 
Entre los rasgos del liderazgo asociados a la dimensión extroversión, se evidenció que en ellas preponderan su calidez, alegría y asertividad - líder de grupo-, los cuales son inherentes al ejercicio del liderazgo compartido que concibe al líder como un servidor, capaz de hacer partícipes a otros para ocupar dicho rol (Raelin, 2005), con el fin de sacar adelante los proyectos de tipo comunitario y participativo característicos de las OSFL (Erazo-Caicedo et al., 2014).

Cabe resaltar que las directoras participantes exhiben puntuaciones altas en todos los rasgos de esta dimensión, lo cual refleja su capacidad de interacción que impacta directamente en la dinamización de los procesos en el interior de las OSFL y en la gestión del ambiente político, con miras a prestar un servicio de excelencia para mejorar la percepción organizacional (Alaimo, 2008), ganar legitimidad con sus grupos de interés y garantizar así aportes y donaciones (Jeong y Kearns, 2015; Suárez, 2007).

Al abordar la correlación entre la extroversión y sus rasgos componentes se encontró que uno de los rasgos que mayor impacto tienen en esta dimensión son las emociones positivas, tal como lo evidencian Diener, Oishi y Lucas, 2003 (como se citan en del Valle, Zamora, Khalil y Altamirano, 2020), al establecer que «existen asociaciones entre la extroversión y la experimentación y expresión de emociones positivas» (p. 58).

Así mismo, Dematteis, Castañeiras y Posada (2013) establecieron que las personas extrovertidas tienen la capacidad de afrontar las situaciones adversas de un modo positivo, gracias a su facilidad para establecer vínculos de confianza — calidez y gregarismo- y a su tendencia a experimentar emociones positivas. De allí la necesidad de que el líder, en este caso las directoras participantes, provean una visión, sean carismáticas, escuchen y generen confianza con el fin de incrementar el desempeño de sus colaboradores (Aragonez y Changanaqui, 2020).

El análisis de la dimensión apertura a nuevas experiencias dio como resultado que las directoras se encuentran entre un nivel medio y alto, lo que significa que son personas sensibles y curiosas, quienes prefieren la novedad a la rutina, lo cual denota que «el individuo 
tiene un amplio rango de intereses, por lo que tiende a vincular sus prioridades con la versatilidad de su entorno al tomar una actitud crítica frente a la sociedad» (Bono y Judge, 2004, como se citan en Arévalo-Avecillas et al., 2019, p. 239).

En relación con los rasgos que abarca esta dimensión se tiene que estas líderes son especialmente sentimentales y curiosas intelectuales, de modo que favorecen la estimulación intelectual de sus seguidores, en el sentido de que los orienten sobre nuevas formas de resolver los problemas (Yukl, 2008); esto redunda en un mejor desempeño organizacional. Así mismo, el enfoque de estas directoras en aspectos concretos, asisten la visión y la coherencia que debe tener un líder transformacional y que debe proyectar en los seguidores, en busca de su compromiso para el logro de los objetivos (Bracho-Parra y García-Guiliany, 2013).

Estas evidencias coinciden con el análisis inferencial realizado, pues algunos de los rasgos del liderazgo que mejor explican la dimensión de apertura son las acciones y las ideas, además de que muestran en las directoras su preferencia por la novedad y su curiosidad intelectual ilimitada, lo cual es ratificado por Peral y Geldenhuys (2020), quienes evidenciaron en su investigación que las personas de mente abierta, creativas y dispuestas a probar nuevas cosas modifican su labor de manera que encaje con sus características personales; esto afecta positivamente el comportamiento en el rol que desempeñan.

Adicionalmente, el coeficiente de Pearson reportó que la apertura a nuevas experiencias es afectada positivamente por el rasgo fantasía, que evidencia la tendencia de las directoras a tener mucha imaginación, lo que se traduce en mayor creatividad. Esta relación es unánimemente confirmada en las investigaciones realizadas por Kaufman y Beghetto (2013), Silvia et al., (2014), Stock, Von Hippel y Gillert (2016), y Kaspi-Baruch (2017), con importantes implicaciones para las organizaciones que quieren ser más creativas, al contratar empleados con rasgos particulares de personalidad como, por ejemplo, la apertura a nuevas experiencias y la extroversión (Jirásek y Sudzina, 2020). 
Los hallazgos en esta dimensión llaman la atención sobre la necesidad de fomentar la creatividad a través de capacitaciones continuas en líderes y colaboradores, con miras a reducir el riesgo de obsolescencia en estas OSFL, que se da no solo por su naturaleza conservadora, sino también por una reducida dinámica innovadora que se traduce en que los proyectos generados no responden a las necesidades de la sociedad (Rey, 2007).

Por su parte, la agradabilidad analizada en las líderes participantes fue una de las dimensiones que obtuvo una mayor calificación. Se destaca su altruismo, tacto, calidez, sencillez y honestidad, lo que refleja una predisposición hacia la cooperación, la cortesía y la valoración de la afiliación mientras evitan el conflicto (Bass y Bass, 2008).

Al estudiar los rasgos de esta dimensión, se encuentra que las directoras destacan en la preocupación por el bienestar de los demás y la disposición a ayudar cuando se requiera, lo cual demuestra que son líderes servidoras que no solo son capaces de brindar atención personalizada, tratar individualmente a cada empleado y desarrollar sus potencialidades (Bass, 1990), sino que generan cambios positivos en la sociedad (Greenleaf, 1995).

Igualmente, se evidencia que las participantes son sinceras, honestas y humildes, rasgos también característicos de las personas que dirigen OSFL y, especialmente, las mujeres, dado que facilitan la aceptación y la interacción con los stakeholders en busca del bien común (Espinosa et al., 2014; Erazo-Caicedo et al., 2014).

Cuando se trata de la correlación entre la agradabilidad y sus rasgos componentes se encuentra que, según el coeficiente de correlación de Pearson, uno de los rasgos que más la afectan positivamente es la confianza, pues está demostrado que quienes son agradables exhiben comportamientos de cooperación y confianza, de modo que son menos propensos a comportarse de manera que dañen a otras personas (Kausel, Leiva, Sanfuentes y Barros, 2012).

Otro de los rasgos que evidencia una fuerte relación con la agradabilidad es el altruismo, tal como lo plantean Lacovella, Díaz-Lázaro y Richard's (2015) cuando encuentran una correlación significativa entre estas dos variables, reafirmando la importancia del fomento 
de este rasgo-valor, especialmente en las mujeres líderes de OSFL, quienes consideran que el liderazgo es ayudar a otros, trabajar con amor y honestidad y no rendirse ante los obstáculos (Moreno et al., 2006).

Los rasgos que integran esta dimensión son deseables en los líderes de las OSFL por sus particularidades y el entorno en el que actúan, en las que se debe promover un liderazgo en el que predomine el respeto, la confianza y la preocupación por el bienestar colectivo (Saravia-Vergara, 2014) y se conciba a los seguidores no como máquinas, sino como personas con necesidades que deben ser motivadas a través del empoderamiento, la capacitación y la atención individual (Osula y Ng, 2014).

Analizando la escrupulosidad se tiene que fue la dimensión que reportó la calificación más alta. Se concluye, entonces, que las directoras reflejan en sus comportamientos la iniciativa, la disciplina, la persistencia, la dedicación y una gran motivación para alcanzar las metas (Zopiatis y Constanti, 2012) lo cual es determinante para generar una cultura de confianza y transparencia en estas OSFL que permita involucrar al equipo de trabajo, quienes perciben un liderazgo auténtico (Rhine, 2015), así como garantizar donaciones de otras fuentes para lograr su perdurabilidad (Hernández, Herrera y Chávez, 2015).

El abordaje de los rasgos que integran la dimensión escrupulosidad permite observar que las directoras son ambiciosas, o sea, que tienen grandes aspiraciones, son analíticas y autodisciplinadas. Estas conductas pueden promover una misión compartida (Mendoza, Escobar y García, 2012) e inspirar a sus colaboradores para lograr las metas (González, González, Ríos y León, 2013).

El análisis inferencial realizado permitió determinar que fueron la deliberación, la competencia y la autodisciplina los rasgos del liderazgo que influyen y mejor explican la escrupulosidad. De allí la relevancia de que las directoras participantes realicen capacitaciones que les posibilite fortalecerse, al ser conscientes de sus propias habilidades y competencias para liderar proyectos, de modo que enriquecen su capacidad de liderazgo (Erazo-Caicedo et al., 2014). 
Lo anterior es demostrado en algunos estudios, como, por ejemplo, el de Hearon y Harrison (2020), quienes evidenciaron que estas características ejercen su influencia sobre cogniciones sociales como la autoeficacia. Así mismo, Morán y Menezes dos Anjos (2016) plantean que la escrupulosidad como tal se relaciona, en primer lugar, con mayor competencia o sensación de capacidad y eficacia, pues los individuos motivados por el logro se consideran mejor preparados para enfrentarse a la vida; en segundo lugar, con un mayor sentido del deber, o sea que se ajustan a sus principios éticos y cumplen con sus obligaciones morales; en tercer lugar, con la deliberación, con lo que demuestran ser reflexivos y prudentes y que tienen en cuenta las consecuencias de sus actos; en cuarto lugar, con la autodisciplina, expresada como la habilidad para terminar las actividades a pesar de los obstáculos, al ser capaces de esforzarse más.

Adicionalmente, Herrera, Al-Lal y Mohamed (2020) encuentran que la escrupulosidad se relaciona con la responsabilidad, la perseverancia, la confiabilidad y la determinación que conlleva un mejor desempeño laboral y académico; además, como lo reafirma Vasada (2012), estas características crean un ambiente de trabajo familiar y amigable que desarrolla una estructura organizacional que soporta la forma de trabajar de la mujer.

Finalmente, es necesario destacar el rol de la autoeficacia en el análisis del liderazgo, en consideración a que los hallazgos encontrados resultan de la percepción de las directoras participantes acerca de los rasgos que, ellas consideran, poseen. Así, una percepción positiva de su capacidad de liderar - autoeficacia - reflejada en algunos de los rasgos estudiados se convierte en un predictor del éxito y en un importante mediador cognitivo de competencia y rendimiento (Gastélum, Guedea, Viciana y Peinado, 2012). Pues cuando estas líderes son conscientes de las capacidades que poseen, pueden mejorar los resultados, lo que corrobora la relación existente entre la autoeficacia y el desempeño laboral (Contreras, Pinto y Sánchez, 2016).

Así mismo, se encuentra que esta autoeficacia de liderazgo está correlacionada de manera directa y significativa con la resiliencia organizacional, que se define como «la capacidad de recuperar el equilibrio después de la exposición a un evento adverso» (Southwick, Charney, 
Martini y Southwick, 2017, como se citan en Rado, 2021, p. 6); y específicamente con dos de sus variables, la capacidad de planeación y de adaptación, determinantes para lograr la sostenibilidad de las OSFL que compiten por recursos escasos y requieren de las continuas contribuciones de sus donantes para cumplir con su objeto social.

\section{Conclusiones}

La contribución de este estudio está enmarcada en dos aspectos fundamentales. El primero es que en el Valle del Cauca no se ha abordado la práctica de estos rasgos del liderazgo, específicamente en este tipo de organizaciones, las cuales requieren de una gerencia con un enfoque multifacético que exhiba competencias en liderazgo, innovación, evaluación crítica, participación, aprendizaje y práctica reflexiva, además de la falta de aplicación de modelos de gestión pensados para el mundo empresarial o la administración pública (Myers, 2004).

El segundo es que no se ha analizado su ejercicio por parte de las mujeres, quienes han jugado tanto en Colombia como en el Valle del Cauca un papel preponderante en este tipo de organizaciones, en los procesos comunitarios y en la construcción de las políticas públicas de equidad de género.

A lo largo de esta investigación fue posible establecer que las dimensiones predominantes en las directoras participantes son la extroversión, la agradabilidad y la escrupulosidad, lo cual concuerda con algunos estudios que plantean que estas predicen el juicio para la toma de decisiones y la inclinación a correr riesgos en muchas situaciones organizacionales (Gullone y Moore, 2000; Markey, Markey, Ericksen y Tinsley, 2006), debido a la autoconfianza y a la sensibilidad que desarrollan las personas que se destacan en estas dimensiones para analizar la información que proviene del entorno (McElroy y Down, 2007).

Las directoras participantes en la investigación consideran, desde su percepción, que tienen la capacidad de: manejar situaciones difíciles, posibilitando superar los obstáculos; interrelacionarse con los grupos de interés para garantizar sus contribuciones; experimentar emociones y sensaciones nuevas con el fin de aceptar los cambios; empatizar las necesidades de los demás, de modo que facilitan su ejercicio del liderazgo; y luchar por sus objetivos a 
partir de la autodisciplina, de manera que sirvan de ejemplo a sus colaboradores para subordinar el interés particular al general.

Así mismo, se puede establecer que algunos de los rasgos preponderantes en las directoras participantes, como, por ejemplo, la confianza y el altruismo - carisma, consideración individualizada - el gregarismo y la asertividad - motivación inspiracional-, y las acciones y las ideas —estimulación intelectual—, favorecen la práctica del liderazgo transformacional, que lleva a mejores resultados en las OSFL (Rowold, Borgmann y Bormann, 2015; SaraviaVergara, 2014; Parra et al., 2010) y ratifican que las mujeres son más transformacionales que los hombres (Bass, 1999; Eagly et al., 2003).

Adicionalmente, se evidencia la necesidad de que algunas líderes se capaciten con miras a fortalecer características tales como la confianza hacia ellas y hacia otras personas, la actividad referida a la rapidez para tomar decisiones y la competencia en relación con su preparación en el ejercicio del liderazgo; lo que les posibilitará incrementar su autoestima, mejorar la percepción y el compromiso de sus colaboradores, así como ser más proactivas para evitar errores y anticipar problemas.

Entre los hallazgos encontrados se resalta que desde la autopercepción de las directoras se aceptan las hipótesis de investigación planteadas en relación con que algunos de los rasgos del liderazgo asociados a las dimensiones neurotismo, extroversión, apertura a nuevas experiencias y escrupulosidad aportan a su variación, exceptuando la dimensión agradabilidad, lo cual podría deberse a su marcado carácter valorativo, lo que ha llevado a no incluirla en muchos modelos factoriales de personalidad (Sánchez y Ledesma, 2019).

Así, los rasgos que mayor impacto tienen en las dimensiones que los integran son la ansiedad social, la actividad, las acciones y la deliberación. De allí la relevancia de fortalecer en las directoras participantes la autoconfianza para evitar los sentimientos de inferioridad, cultivar la creatividad a fin de aumentar la flexibilidad y la inteligencia emocional para manejar las emociones y el pensamiento crítico, con el propósito de moderar las reacciones ante las situaciones que se presentan, en consideración a que las mujeres son más 
conscientes de sus propias emociones y de las de los demás con respecto a los hombres (Zarate y Matviuk, 2012).

Estos resultados, producto de la autopercepción de las líderes participantes, dan cuenta de la importancia de la autoeficacia como predictora del éxito, pues el hecho de que ellas sean conscientes de sus capacidades les permite no solo mejorar su gestión, sino también acrecentar el capital psicológico de sus colaboradores (Contreras et al., 2016).

Las OSFL surgen debido a la incapacidad del Estado de satisfacer las necesidades básicas de la población, que pasó de ser un Estado del bienestar que realizaba inversión social a un Estado neoliberal, cuyos fondos están destinados, en mayor medida, a responder a la dinámica que le impone el mercado (Schvarstein, 2003). Por esta razón, la investigación de la dinámica organizacional de las OSFL debe convertirse en un tema de interés, debido no solo a la escasez de estudios empíricos, sino a la relevancia de este tipo de organizaciones, en razón al rol que desempeñan en la sociedad, al trabajar donde fallan los mercados y el Estado (Weisbrod, 1975).

Así, el liderazgo en las OSFL debe caracterizarse por una influencia ejercida a partir de valores como la honestidad y la integridad, y por comportamientos que promuevan la colaboración, el empoderamiento, la comunicación y la construcción de confianza; además, según numerosos estudios, puede darse a partir de la práctica del liderazgo transformacional, el cual, se ha demostrado, impacta de una forma positiva en el desempeño de los empleados y en la eficacia de las organizaciones.

Ahora bien, se hace referencia al liderazgo transformacional, pues muchas de sus características coinciden con los rasgos del liderazgo aquí analizados a partir del inventario revisado de personalidad NEO PI-R, tal como lo plantean Arévalo-Avecillas et al. (2019) al evidenciar en su investigación que los dominios extroversión, apertura a nuevas experiencias y escrupulosidad tienen una relación positiva y significativa estadísticamente con el estilo de liderazgo transformacional. Esto quiere decir que «las personas con altos niveles de dichos 
rasgos son más propensas a presentar comportamientos de líderes transformacionales» (p. 247).

Así mismo, en la investigación realizada por Contreras (2008) se encuentra que el estudio de la personalidad del líder a partir de este modelo de los cinco factores o inventario de la personalidad es importante, en la medida en que permite no solo predecir ciertas actitudes y características relacionadas con el liderazgo, tales como satisfacción laboral, creatividad y toma de decisiones (Malouff et al., 2006), sino identificar potenciales líderes por sus características de personalidad.

A partir de lo anterior, se concluye la importancia de la formación de los líderes de las OSFL a través de programas de educación formal y no formal que desarrollen habilidades y capacidades como la comunicación, la administración y la gestión, la recaudación de fondos, el desarrollo del voluntariado, la dedicación o compromiso con la misión de la organización y la ética, para lograr la consecución de recursos y la generación de identidad (Ahmed, 2005; Jiménez, 2012).

Por otra parte, la construcción social dominante por género es evidente en el trabajo que realizan las directoras, pues todas ellas tuvieron como principal motivación ayudar y cuidar a otros, afirmando así el hecho social de que a las mujeres, por ser mujeres, les compete el ser las proveedoras de los afectos; sin embargo, en este análisis se corrobora cómo estas líderes, en su mayoría fundadoras de sus instituciones, han logrado ejercer su papel tradicional como cuidadoras ya no solo en el ámbito privado-doméstico, sino también en el ámbito público, lo cual implica modificaciones en la forma de asumir la división sexual del trabajo que impactan en la concepción del género.

La reflexión anterior da cuenta del cumplimiento del objetivo de esta investigación en relación con la determinación de los rasgos del liderazgo preponderantes en las directoras participantes y su influencia en la variación de la dimensión de la personalidad que los integra, con el fin de predecir su potencial de liderazgo y analizar, con base en los estudios 
empíricos referentes, su incidencia en el desempeño de los colaboradores y de la organización.

Las limitaciones del estudio serían, en primer lugar, que el análisis se realizó con un número limitado de organizaciones, lo cual repercute en la validez y la confiabilidad de los datos obtenidos; en segundo lugar, la aplicación de la encuesta utilizada en esta investigación solo se realizó a una sola persona, en este caso, la directora de la fundación, debido, principalmente, a la disponibilidad de tiempo. Así, hubiera sido recomendable contar con la opinión de diferentes públicos, es decir, no solo de las líderes, sino también de sus colaboradores y otros grupos, como, por ejemplo, los beneficiarios de las fundaciones, con el fin de disminuir al máximo el sesgo en la información obtenida.

La intención es que este estudio contribuya a la academia, en especial a las áreas de investigación en liderazgo, género y organizaciones sin fines de lucro, a los estudiantes que estén interesados en conocer acerca del liderazgo femenino y la dinámica de las OSFL, así como a los dueños o gerentes de empresas que quieran potenciar el liderazgo en su talento humano, especialmente en las mujeres, pues como lo evidencian Sen y Metzger (2010), la globalización ha planteado la necesidad de instaurar en las empresas estilos de liderazgo más colaborativos, en los que se promueva la construcción de redes y el trabajo en equipo, a lo que las mujeres han respondido efectivamente no solo adoptando este estilo, sino apropiándolo para generar mayor rentabilidad a la organización.

Finalmente, esta investigación se convierte en una motivación para desarrollar posteriores proyectos que permitan analizar otros tipos de liderazgo en las mujeres, como, por ejemplo, el transformacional, el servidor, el visionario o el social, así como validar el instrumento propuesto en otros líderes de organizaciones con fines de lucro e incorporar en el análisis las percepciones de otros grupos de interés, tales como el Gobierno, los beneficiarios y los donantes. 


\section{Referencias}

Ahmed, S. (2005). Desired competencies and job duties of non-profit CEOs in relation to the current challenges: through the lens of CEOs' job advertisements. Journal of Management Development, 24(10), 913-928. DOI: https://doi.org/10.1108/02621710510627055

Akinwale, 0. (2019). Employee voice: speaking up in organisation as a correlate of employee productivity in oil and gas industry-an empirical investigation from Nigeria. Serbian Journal of Management, 14(1), 97-121. DOI: https://doi.org/10.5937/sjm14-15308

Alaimo, S. (2008). Nonprofits and evaluation: managing expectations from the leader's perspective. New Directions for Evaluation, (119), 73-92. DOI: https://doi.org/10.1002/ev.269

Alcaldía de Bogotá. (2014). Sistema de información de personas jurídicas: definiciones. Recuperado de https://bit.ly/3B1gyOF

Aragonez, J.; Changanaqui, A. (2020). Liderazgo transformacional y su relación con la productividad de los trabajadores en las empresas de servicios de auditoría en Lima Metropolitana, 2019 (Tesis de pregrado). Universidad Peruana de Ciencias Aplicadas. Lima, Perú. Recuperado de https://bit.ly/3uiRmR9

Arévalo-Avecillas, D.; Padilla-Lozano, C.; Pino, R.; Cevallos, H. (2019). Los dominios de la personalidad y su relación con el estilo de liderazgo transformacional. Información Tecnológica, 30(3), 237248. DOI: https://doi.org/10.4067/S0718-07642019000300237

Bass, B. (1990). From transactional to transformational leadership: learning to share the vision. Organizational Dynamics, 18(3), 19-31. DOI: https://doi.org/10.1016/0090-2616(90)90061$\underline{\mathrm{S}}$

Bass, B. (1999). Two decades of research and development in transformational leadership. European Journal of Work and Organizational Psychology, 8(1), 9-32. DOI: https://doi.org/10.1080/135943299398410

Bass, B.; Bass, R. (2008). The bass handbook of leadership: theory, research, and managerial applications (4a ed.). Nueva York: Free Press.

Bennis, W. (1977). Managing the dream: leadership in the 21st Century. Journal of Organizational Change Management, 2(1), 6-10. DOI: https://doi.org/10.1108/09534818910134040

Blake, R.; Mouton, J. (1967). The managerial grid in three dimensions. Trainig and Development Journal, 21(1), 2-5.

Bracho-Parra, 0.; García-Guiliany, J. (2013). Algunas consideraciones teóricas sobre el liderazgo transformacional. Telos. Revista de Estudios Interdisciplinarios en Ciencias Sociales. 15(2), 165177.

Burns, J. (1978). The power of vision. Recuperado de https://bit.ly/3udUSw0 
Butler, J. (1998). Actos performativos y constitución del género: un ensayo sobre fenomenología y teoría feminista. Debate Feminista, https://doi.org/10.22201/cieg.2594066xe.1998.18.526

18, 296-314. DOI:

Cárdenas, C. (2002). La nueva gerencia. Revista Dinero.

Carlyle, T. (1904). On heroes, hero-worship and the heroic in history. Londres: Chapman and Hall.

Chodorow, N. (1978). The reproduction of mothering: psychoanalysis and the sociology of gender. Berkeley: University of California Press. DOI: https://doi.org/10.1525/9780520924086

Cohen, W. (2011). Absolute integrity is the basis of heroic leaders. Leader to Leader, (59), 46-51. DOI: https://doi.org/10.1002/ltl.455

Contreras, F. (2008). Liderazgo: perspectivas de desarrollo e investigación. International Journal of Psychological Research, 1(2), 64-72. DOI: https://doi.org/10.21500/20112084.923

Contreras, I.; Pinto, L.; Sánchez, M. (2016). Liderazgo y autoeficacia en el comportamiento organizacional positivo (Tesis de pregrado). Universidad del Rosario. Bogotá, Colombia. Recuperado de https://bit.ly/2XWZE5v

Costa, P. T.; McCrae, R. R. (1992). NEO PI-R professional manual. Odesa: Psychological Assesment Resources.

Cowley, W. (1931). Three distinctions in the study of leaders. The Journal of Abnormal and Social Psychology, 26(3), 304-313. DOI: https://doi.org/10.1037/h0074766

D’Alessio, F. (2010). Liderazgo y atributos gerenciales: una visión global y estratégica. México: Prentice Hall.

del Valle, M.; Zamora, E.; Khalil, Y.; Altamirano, M. (2020). Rasgos de personalidad y dificultades de regulación emocional en estudiantes universitarios. Psicodebate, 20(1), 56-67. DOI: https://doi.org/10.18682/pd.v20i1.1877

Dematteis, M.; Castañeiras, C.; Posada, M. (2013). Evaluación de la personalidad como recurso de salud. Anuario de Proyectos e Informes de Becarios de Investigación, (10), 861-867. Recuperado de https://bit.ly/3oeZ65w

Do, M.; Minbashian, A. (2014). A meta-analytic examination of the effects of the agentic and affiliative aspects of extraversion on leadership outcomes. The Leadership Quarterly, 25(5), 1040-1053. DOI: https://doi.org/10.1016/j.leaqua.2014.04.004

Dubrin, A. J. (2008). Relaciones humanas, comportamiento humano en el trabajo (9a ed.). México: Pearson Educación.

Eagly, A. H.; Karau, S. J. (2002). Role congruity theory of prejudice toward female leaders. Psychological Review, 109, 573-598. DOI: https://doi.org/10.1037/0033-295X.109.3.573 
Eagly, A.; Johannesen-Schmidt, M.; Engen, M. van. (2003). Transformational, transactional, and laissez-faire leadership styles: a meta-analysis comparing women and men. Psychological Bulletin, 129(4), 569-591. DOI: https://doi.org/10.1037/0033-2909.129.4.569

Erazo-Caicedo, M.; Jiménez-Ruiz, M.; López-Morales, C. (2014). Empoderamiento y liderazgo femenino; su papel en la autogestión comunitaria en el corregimiento El Hormiguero-Valle del Cauca. Avances en Psicología Latinoamericana, 32(1), 149-157. DOI: https://doi.org/10.12804/apl32.1.2014.10

Espinosa, J.; Contreras, F.; Uribe, A.; Barbosa, D. (2014). Patrones de liderazgo en la cultura regional: un análisis intersectorial. Universidad \& Empresa, 16(27), 53-69. DOI: https://doi.org/10.12804/rev.univ.empresa.27.2014.01

Fiedler, F.; Ayman, R.; Chemers, M. (1995). The contingency model of leadership effectiveness: its levels of analysis. Leadership Quarterly, 6(2), 147-167. DOI: https://doi.org/10.1016/10489843(95)90032-2

Fleishman, E.A. (1972). Manual for the supervisory behavior description questionnaire. Washington, D.C.: American Institutes for Research.

Gastélum, G.; Guedea, J.; Viciana, J.; Peinado, J. (2012). Composición factorial de una escala de autoeficacia en el ámbito del trabajo en equipo y liderazgo en universitarios de ciencias de la salud. Formación Universitaria, 5(4), 49-60. DOI: https://doi.org/10.4067/S0718$\underline{50062012000400006}$

Gibson, J.; Ivancevich, J.; Donnelly, J. (2001). Las organizaciones: comportamiento, estructura, procesos (10 ed ed.). Santiago de Chile: McGraw-Hill Interamericana.

González, O.; González, O.; Ríos, G.; León, J. (2013). Características del liderazgo transformacional presentes en un grupo de docentes universitarios. Telos, 15(3), 355-371.

González-Villalobos, J.; Marrero, R. (2017). Determinantes sociodemográficos y personales del bienestar subjetivo y psicológico en población mexicana. Suma Psicológica, 24(1), 59-66. DOI: https://doi.org/10.1016/j.sumpsi.2017.01.002

Greenleaf, R. (1995). Servant leadership. En J. T. Wren (Ed.) The Leader's Companion: Insights on Leadership Through the Ages Paulist Press. (18-23). Nueva York: The Free Press. Recuperado de https://bit.ly/3m2ZqSe

Gullone, E.; Moore, S. (2000). Adolescent Risktaking and the Five-factor Model of Personality. Journal of Adolescence, 23(4), 393-407. DOI: https://doi.org/10.1006/jado.2000.0327

Hall, R. (1983). Organizaciones, estructura y proceso (3a ed.). Madrid: Prentice Hall.

Halpin, A.W. (1957). Manual for the leader behavior description questionnaire. Columbus: The Ohio State University, Bureau of Business Research. 
Hearon, B.; Harrison, T. (2020). Not the exercise type? Personality traits and anxiety sensitivity as predictors of objectively measured physical activity and sedentary time. Journal of Health Psychology. DOI: https://doi.org/10.1177\%2F1359105320906242

Hernández, J.; Herrera, F.; Chávez, C. (2015). Capacidades, liderazgos y estrategias de gestión de organizaciones de la sociedad civil en zonas rurales. Contaduría y Administración, 60(4), 817835. DOI: https://doi.org/10.1016/j.cya.2015.07.001

Hernández, R.; Fernández, C.; Baptista, P. (2010). Metodología de la investigación (5ae ed.). Ciudad de México: McGraw Hill.

Herrera, L.; Al-Lal, M.; Mohamed, L. (2020). Academic achievement, self-concept, personality and emotional intelligence in primary education. Analysis by gender and cultural group. Frontiers in Psychology, 10, 3075. DOI: https://doi.org/10.3389/fpsyg.2019.03075

Hersey, P.; Blanchard, K.; Natemeyer, W. (1979). Situational leadership, perception and the impact of power. Group and Organization Studies, 4(4), 418-428. DOI: https://doi.org/10.1177/105960117900400404

House, R. (1992). Charismatic leadership in service-producing organizations. International Journal of Service Industry Management, 3(2), 5-16. DOI: https://doi.org/10.1108/09564239210011944

Jeong, B.; Kearns, K. (2015). Accountability in Korean NPOs: perceptions and strategies of NPO leaders. Voluntas: International Journal of Voluntary and Nonprofit Organizations, 26(5), 19752001. DOI: https://doi.org/10.1007/s11266-014-9492-8

Jiménez, 0. (2012). El papel del liderazgo en Colombia desde la perspectiva de la valoración del ambiente laboral: un estudio aplicado de Great Place to Work en el periodo 2009-2011 (Tesis de pregrado). Universidad Ean. Bogotá, Colombia. Recuperado de https://bit.ly/3AMdIgb

Jirásek, M.; Sudzina, F. (2020). Big five personality traits and creativity. Quality Innovation Prosperity/Kvalita Inovácia Prosperita, 24(3), 90-105. DOI: https://doi.org/10.12776/qip.v24i3.1509

Kaspi-Baruch, O. (2017). Big five personality and creativity: the moderating effect of motivational goal orientation. The Journal of Creative Behavior, 53(3), 325-338. DOI: https://doi.org/10.1002/jocb.183

Katz, R.; Kahn, D. (1978). The social psychology of organizations (2a ed). Nueva York: Wiley.

Kaufman, J.; Beghetto, R. (2013). Do people recognize the four Cs? Examining layperson conceptions of creativity. Psychology of Aesthetics, Creativity, and the Arts, 7(3), 229-236. DOI: https://doi.org/10.1037/a0033295

Kausel, E.; Leiva, P.; Sanfuentes , M.; Barros , E. (2012). Más allá de los cinco grandes: disposiciones y personalidad en la predicción de decisiones deshonestas en el contexto organizacional. Innovar Journal Revista de Ciencias Administrativas y Sociales, 22(44), 40-60. 
Kergoat, D. (2002). División sexual del trabajo y relaciones sociales entre los sexos. En E. Hirata; F. Laborie; H. Le Doaré; D. Senotier (Coord.) Diccionario crítico del feminismo. (66-75). Madrid: Editorial Síntesis.

Lacovella, J.; Díaz-Lázaro, C.; Richard's, M. (2015). Relación entre la empatía y los cinco grandes factores de personalidad en una muestra de estudiantes universitarios. Revista Argentina de Ciencias del Comportamiento, 7(2), 14-21. Recuperado de https://bit.ly/3icbYWh

Lamas, M. (1996). La perspectiva de género. Revista de Educación y Cultura de la Sección 47 del SNTE, (8), 1-10. Recuperado de https://bit.ly/3zGTm6N

Lamas, M. (2000). Diferencias de sexo, género y diferencia sexual. Cuicuilco, 7(18), 1-25.

Lamas, M. (2008). ¿A dónde va la antropología? Complejidad y claridad en torno al concepto género. Ciudad de México: División de Ciencias Sociales y Humanidades de la UAM - Iztapalapa. Recuperado de https://bit.ly/3obKSSO

Lewin, K.; Lippitt, R.; White, R. (1939). Patterns of aggressive behavior in experimentally created «social climates». The Journal of Social Psychology, 10(2), 271-299. DOI: https://doi.org/10.1080/00224545.1939.9713366

Limura, S.; Taku, K. (2018). Gender differences in relationship between resilience and big five personality traits in Japanese adolescents. Psychological Reports, 121(5), 920-931. DOI: https://doi.org/10.1177/0033294117741654

Lussier, R.; Achua, C. (2005). Liderazgo. México: Thomson.

Malouff, J. M.; Thorsteinsson, E. B.; Schutte, N. S. (2006). The Five-factor Model of Personality and Smoking: A Meta-analysis. Journal of Drug Education, 36, 47-58. DOI: https://doi.org/10.2190/9ep8-17p8-ekg7-66ad

Markey, C. N.; Markey, P. M.; Ericksen, A. J.; Tinsely, B. J. (2006). Children's Behavioral Patterns, the Five-Factor Model of Personality, and Risk Behaviors. Personality and Individual Differences, 41, 1503-1513. DOI: https://doi.org/10.1016/j.paid.2006.06.007

McElroy, T.; Dowd, K. (2007). Susceptibility to anchoring effects: How openness-to-experience influences responses to anchoring cues. Judgment and Decision Making, 2(1), 48-53.

Mejía-Londoño, E.; Zea-Jaramillo, A.; Pérez-Ortega, G. (2004). Caracterización de los estilos de liderazgo en algunas ONG ambientales en Antioquia. Dyna, 71(143), 13-23.

Mendoza, I.; Escobar, G.; García, B. (2012). Influencia del liderazgo transformacional en algunas variables de satisfacción organizacional en personal docente y administrativo de una institución pública de educación media superior. Revista del Centro de Investigación. Universidad La Salle, 10(38), 189-206. 
Morán, C.; Menezes dos Anjos, E. (2016). La motivación de logro como impulso creador de bienestar: su relación con los cinco grandes factores de la personalidad. International Journal of Developmental and Educational Psychology INFAD Revista de Psicología, 2(1), 31-40. DOI: https://doi.org/10.17060/ijodaep.2016.n1.v2.292

Moreno, G.; Badillo, A.; Marín, A. (2006). Participación y liderazgo de las mujeres en organizaciones de población en situación de desplazamiento forzado por la violencia. Bucaramanga: Centro de Estudios de Género Magdalena León de la Fundación Mujer y Futuro. Recuperado de https://bit.ly/39HfNhp

Myers, J. (2004). Developing managers: a view from the non-profit sector. Journal of European Industrial Training, 28(8/9), 639-656. DOI: https://doi.org/10.1108/03090590410566561

Nicholson, N. M.; Fenton-O'Creevy, M.; Soane, E.; Willman, P. (2005). Personality and DomainSpecific Risk Taking. Journal of Risk Research, 8(2), 157-176. DOI: https://doi.org/10.1080/1366987032000123856

Osula, B.; Ng, E. (2014). Toward a collaborative, transformative model of non-profit leadership: some conceptual building blocks. Administrative Sciences, 4(2), 87-104. DOI: https://doi.org/10.3390/admsci4020087

Pardo, A.; Ruiz, M. (2005). Análisis de datos con SPSS 13 Base. Madrid: McGraw Hill.

Parra, G.; Useche, L.; Barbosa, L. (2010). Liderazgo e inteligencia emocional en personas que ocupan jefaturas en una Institución que presta servicios de salud ubicada en Bogotá (Tesis de pregrado). Pontificia Universidad Javeriana. Bogotá, Colombia. Recuperado de https://bit.ly/3EZe0CM

Peral, S.; Geldenhuys, M. (2020). The indirect relationship between personality and performance through job crafting behaviour. SA Journal of Industrial Psychology, 46, a1715. DOI: https://doi.org/10.4102/sajip.v46i0.1715

Rado, E. (2021). Resiliencia organizacional y autoeficacia de liderazgo en el centro de salud de Independencia, Cusco 2020 (Tesis de maestría). Universidad Cesar Vallejo. Lima, Perú. Recuperado de https://bit.ly/3i9fo]9

Raelin, J. (2005). We the leaders: in order to form a leaderful organization. Journal of Leadership and Organizational Studies, 12(2), 18-30. DOI: https://doi.org/10.1177/107179190501200202

Rey, M. (2007). La gestión de las fundaciones en el siglo XXI: retos, tendencias y una hoja de ruta. Revista Española del Tercer Sector, (6), 37-54.

Rhine, A. (2015). An examination of the perceptions of stakeholders on authentic leadership in strategic planning in Nonprofit Arts Organizations. The Journal of Arts Management, Law, and Society, 45(1), 3-21. DOI: https://doi.org/10.1080/10632921.2015.1013169

Robbins, S.; Coulter, M. (2005). Administración (8ae ed.). México: Pearson Educación. 
Rowold, J.; Borgmann, L.; Bormann, K. (2015). Which leadership constructs are important for predicting job satisfaction, affective commitment, and perceived job performance in profit versus nonprofit organizations? Nonprofit Management \& Leadership, 25(2), 147-164. DOI: https://doi.org/10.1002/nml.21116

Rubin, G. (1986). El tráfico de mujeres: notas sobre la «economía política» del sexo. Nueva Antropología, 8(30), 95-145. Recuperado de https://bit.ly/3EZkthh

Sánchez, R.; Ledesma, R. (2019). Los cinco grandes factores: cómo entender la personalidad y cómo evaluarla. En A. Monjeau (Ed.) Conocimiento para la transformación. (131-160). Buenos Aires: Ediciones Universidad Atlántida.

Saravia-Vergara, E. (2014). Caracterización de estilos de liderazgo mediante el análisis de redes sociales. Journal of Business, 6(1), 30-42. DOI: https://doi.org/10.21678/jb.2014.65

Schein, V.; Mueller, R.; Lituchy, T.; Liu, J. (1996). Think manager-think male: a global phenomenon? Journal of Organizational Behavior, 17(1), 33-41. DOI: https://doi.org/10.1002/(SICI)10991379(199601)17:1<33::AID-JOB778>3.0.CO;2-F

Schriesheim, Ch.; Stogdill, R. (1975). Differences in factor structure across three versions of the Ohio State leadership scales. Personnel Psychology, 28(2), 189-206. DOI: https://doi.org/10.1111/j.1744-6570.1975.tb01380.x

Schvarstein, L. (2003). La inteligencia social de las organizaciones: desarrollando las competencias necesarias para el ejercicio efectivo de la responsabilidad social. Buenos Aires: Paidós.

Scott, J. (2003). El género: Una categoría útil para el análisis histórico. En M. Lamas (Comp.) El género: la construcción cultural de la diferencia sexual. (265-302). Ciudad de México: UNAM. Recuperado de https://bit.ly/3CWagAl

Sen, A.; Metzger, J. (2010). Women leadership and global power: evidence from the United States and Latin America. International Journal of Management and Marketing Research, 3(2), 75-84. Recuperado de https://bit.ly/2WkvjwM

Silvia, P.; Beaty, R.; Nusbaum, E.; Eddington, K.; Levin-Aspenson, H.; Kwapil, T. (2014). Everyday creativity in daily life: An experience-sampling study of «little c» creativity. Psychology of Aesthetics, Creativity, and the Arts, 8(2), 183-188. DOI: https://doi.org/10.1037/a0035722

Stock, R.; Von Hippel, E.; Gillert, N. (2016). Impacts of personality traits on consumer innovation success. Research Policy, 45(4), 757-769. DOI: https://doi.org/10.1016/j.respol.2015.12.002

Stogdill, R. (1963). Manual for the leader behavior description questionnaire - form XII. Columbus: The Ohio State University.

Stogdill, R.; Bass, B. (1990). Bass \& Stogdill's handbook of leadership: theory, research, and managerial applications. Nueva York: The Free Press. 
Suárez, J. (2007). Relaciones entre organizaciones y stakeholders: necesidad de una interacción mutua entre los diversos grupos de interés. INNOVAR: Revista de Ciencias Administrativas y Sociales, $17(30), 153-158$.

Tannenbaum, R.; Schmidt, W. (mayo de 1973). How to choose a leadership pattern. Harvard Business Review. Recuperado de https://bit.ly/3EXbJIp

Vasada, T. (2012). A cultural feminist perspective on leadership in nonprofit organizations: a case of women leaders in India. Public Administration Quarterly, 36(4), 462-503.

Vivas-Cortés, O.; González-Tobito, J.; Gómez-Sarmiento, J. (2015). Caracterización de las diferentes formas de organización social en Colombia: las organizaciones solidarias de desarrollo (OSD). Revista Científica General José María Córdova, 13(13), 57-79. DOI: https://doi.org/10.21830/19006586.31

Weber, E.; Blais, A.; Betz, N. E. (2002). A Domainspecific Risk-attitude Scale: Measuring Risk Perceptions and Risk Behaviors. Journal of Behavioral Decision Making, 15(4), 263-290. DOI: https://doi.org/10.1002/bdm.414

Weisbrod, B. (1975). Toward a theory or the voluntary nonprofit sector in a three-sector economy. En S. Phelps (Ed.) Altruism, morality, and economic theory. (171-196). Nueva York: Rusell Sage Foundation.

Yukl, G. (2008). Liderazgo en las organizaciones. Madrid: Prentice Hall.

Zaleznik, A. (1990). The leadership gap. The Executive, 4(1), 7-22. DOI: https://doi.org/10.5465/ame.1990.4274698

Zárate, R.; Matviuk, S. (2012). Inteligencia emocional y prácticas de liderazgo en las organizaciones colombianas. Cuadernos de Administración, 28(47), 89-102.

Zopiatis, A.; P. Constanti. (2012). Extraversion, openness and conscientiousness: The route to transformational leadership in the hotel industry. Leadership \& Organization Development Journal, 33(1), 86-104. DOI: https://doi.org/10.1108/01437731211193133 